\title{
Delayed Asthmatic Response to Allergen Challenge and Cytokines Released by Nonspecifically Stimulated Blood Cells
}

\author{
Zdenek Pelikan \\ Allergy Research Foundation, Effenseweg 42, 4838 BB Breda, The Netherlands \\ Correspondence should be addressed to Zdenek Pelikan; zpelikan@casema.nl
}

Received 9 October 2012; Accepted 3 December 2012

Academic Editors: A. El-Shazly, D. R. Getts, J. Meseguer, and M. Reale

Copyright ( 2013 Zdenek Pelikan. This is an open access article distributed under the Creative Commons Attribution License, which permits unrestricted use, distribution, and reproduction in any medium, provided the original work is properly cited.

\begin{abstract}
Background. Bronchial asthma patients can develop various asthmatic response types following bronchial allergen challenge, such as immediate (IAR), late (LAR), dual late (DLAR), or delayed (DYAR), due to different immunologic mechanisms. The DYAR, recorded in 24 patients, beginning between 26 and $32 \mathrm{hrs}$ and lasting up to $56 \mathrm{hrs}$ after the bronchial allergen challenge, differs from the IAR, LAR, and DLAR in clinical, diagnostic, and immunologic aspects. Objective. To investigate amounts of particular cytokines released by the blood cells after an additional nonspecific stimulation with Phorbol 12-myristate 13-acetate (PMA) during the DYAR. Methods. In 24 patients, the repeated DYAR was supplemented with determination of cytokines both in the nonstimulated plasma and in the supernatants of the blood cells stimulated with PMA before and up to 72 hours after the bronchial challenge, by means of enzyme-linked immunoassay. Results. No significant changes of the prechallenge cytokine concentrations in the non-stimulated serum were recorded in the DYAR patients as compared with the healthy subjects. The DYAR was accompanied by significantly increased postchallenge concentrations $(P<0.05)$ of IL-2, IL-8, IL-12p70, IL-13, IL-18, IFN- $\gamma$, G-CSF, TNF- $\alpha$, and TGF- $\beta$, while decreased concentration of IL-7 $(P<0.05)$ in the nonstimulated plasma. The significantly increased postchallenge concentrations of IL-2, IL-8, IL-12p70, IL-13, IL-18, IFN- $\gamma$, TNF- $\alpha$, and TGF- $\beta$ were released by peripheral blood cells after stimulation with PMA, as compared with both their prechallenge concentrations and with the PBS control values. Conclusions. These results would support evidence for an important role of the Th1 cells, neutrophils, monocytes, and probably also NK cells in the immunologic mechanism(s) leading to the development of the clinical DYAR. Nevertheless, an additional role of macrophages, endothelial and epithelial cells in these mechanisms cannot be even excluded.
\end{abstract}

\section{Introduction}

Allergic bronchial asthma is a multifaceted disorder, where various immunologic mechanisms can participate [1-8]. The causal role of immediate hypersensitivity including IgE antibodies, mast cells, eosinophils, and $\mathrm{Th}_{2}$ lymphocytes, in this disorder has been established [1-11]. Knowledge of the role of other hypersensitivity mechanisms in this condition remains still deficient $[1-4,7-18]$.

Bronchial asthma patients can respond to allergen challenge by various types of asthmatic response, such as immediate (IAR), late (LAR), or dual late (DLAR) responses $[5,7$, $8,10,11,18-23$ ], having been investigated extensively from various clinical, immunologic and pharmacologic points of view $[5,7-39]$.

In some patients examined at our clinic, an unusual asthmatic response appearing 26-56 hours after the bronchial challenge with inhalant allergens was recorded [40, 41]. This response, displaying clinical and immunologic features different from those of the IAR and of the LAR, was designated as "delayed asthmatic response" (DYAR) [40, 41].

Cytokines representing very important components of the immunologic mechanism(s) execute a number of intracellular, extracellular, and intercellular activities, such as stimulating, inhibiting, and chemotactic effects as well as signal transmission among various cell types $[1,2,6,17,18,21$ 33]. From this point of view, the cytokines are closely related to various cell types participating on various levels and during various stages of the immunologic events. This relation has two basic aspects: a morphologic aspect concerning the production of particular cytokines by certain cell types and a functional aspect concerning the specific effects of particular cytokines on various target cell types $[1,2,6]$. The cellular 


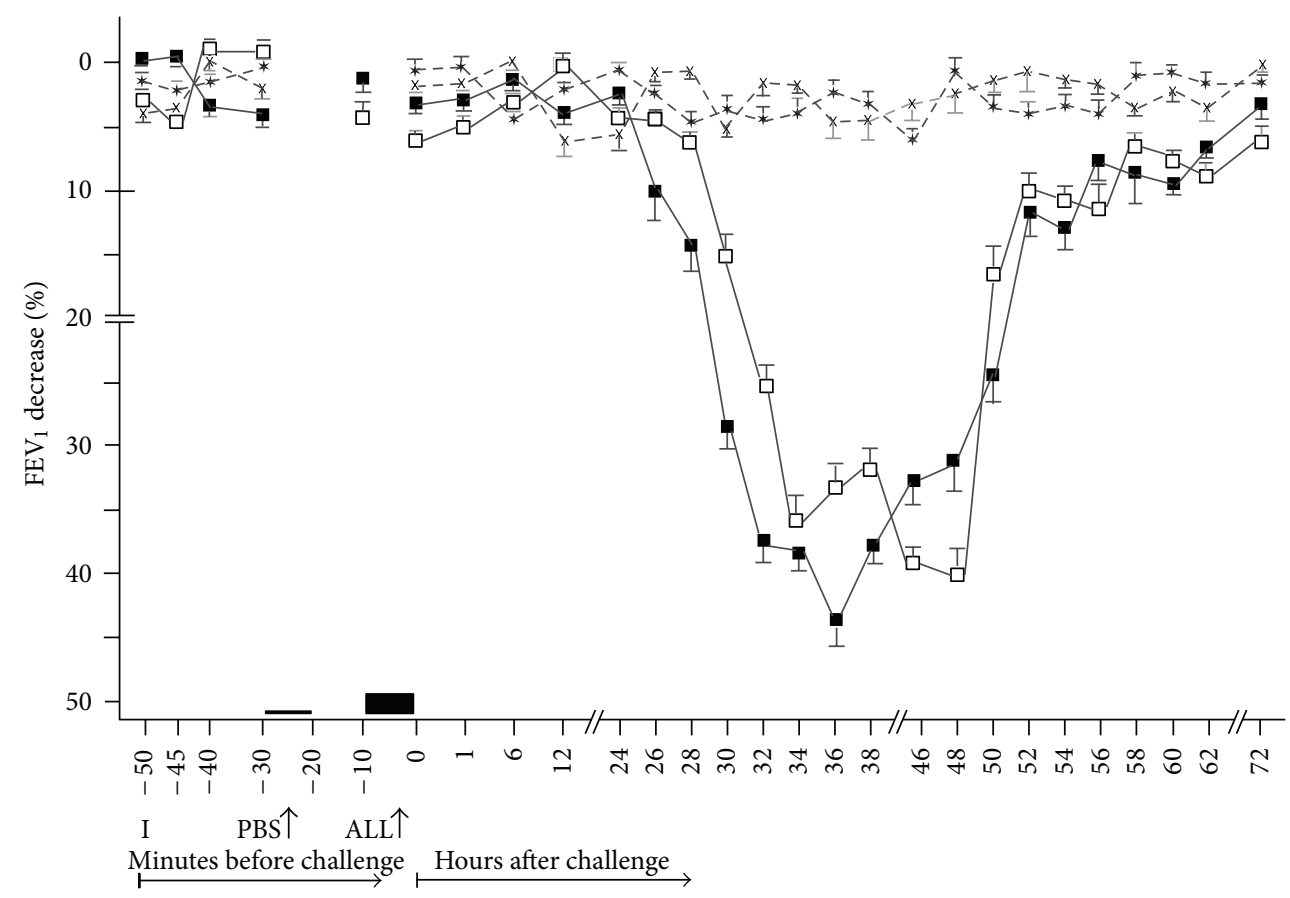

FIGURE 1: Delayed asthmatic response to allergen challenge (DYAR) and phosphate buffered saline (PBS) control challenge. The mean percentage changes in the $\mathrm{FEV}_{1}$ values calculated from 24 DYARs and 24 PBS control challenges; $(\square)$ : the initial DYAR; ( $\square$ ): the repeated DYAR; (*): the initial PBS; $(\times)$ : the repeated PBS; I: initial (baseline) values; ALL: allergen challenge; PBS: phosphate buffered saline; Bars: means \pm SEM.

sources of particular cytokines, being a very complex and continuously updated topic, are actually outside the scope of this study and are presented in detail in a number of excellent reviews [6].

The purpose of this study was to investigate: (1) the intracellular cytokine potential which can be released after the "in vitro" nonspecific stimulation of the blood cell in patients developing the DYAR in comparison with the patients demonstrating IAR, LAR, or DLAR as well as with healthy subjects; (2) the ratio between the concentrations of cytokines released into the peripheral blood "in vivo" during the DYAR and the amounts of intracellular cytokines released by "in vitro" nonspecific cell stimulation; (3) the activation degree of the particular blood cell types during the DYAR and that caused by the "in vitro" nonspecific stimulation.

\section{Material and Methods}

2.1. Patients. Twenty-four patients with bronchial asthma examined at our Department of Allergology and Immunology, Inst. Med. Sci “De Klokkenberg”, Breda, The Netherlands), and developing DYAR (Figure 1) after the bronchial allergen challenge (BPT), volunteered to participate in this study.

These patients, 21-48 years of age, demonstrating reversible bronchial constriction alternating with symptomfree periods, showed pulmonary function without any restrictive changes (Table 1). They had no airway infections and did not use oral corticosteroids or immunotherapy. They were examined by routine diagnostic procedure, serving also as an inclusion-exclusion criteria, consisting of various diagnostic parameters (Table 2) and including also 43 BPTs with inhalant allergens (Table 3) and 24 PBS control challenges. All BPTs were performed in a period without manifest bronchial complaints, outside the allergen-relevant season and during hospitalization.

Inhalation corticosteroids $(n=6)$ and long-acting $\beta_{2}$ sympathomimetics $(n=8)$ were withdrawn 4 weeks, cromolyn $(n=3)$, nedocromil sodium $(n=5)$ and leukotrieneantagonists $(n=2) 2$ weeks, and other treatments 48 hours prior the BPTs. If the postchallenge $\mathrm{FEV}_{1}$ values decreased with $50 \%$ or more with respect to the predicted values, the patients were treated with inhalation of $200-400 \mathrm{mcg}$ Salbutamol.

In the DYAR patients and all control subjects, a single determination of various cytokines both in the nonstimulated serum (Table 4) and those released from peripheral blood cells after an "in vitro" stimulation with Phytohemagglutinin (PHA), Phorbol 12-myristate 13-acetate (PMA), and Nformylmethionyl-leucyl-phenylalanine (fMLP) (Table 5) was performed.

In the DYAR patients, the BPTs and PBS controls were repeated 2-6 weeks later. The repeated DYARs and PBS controls (Figure 1) were supplemented with recording of the cytokines in the nonstimulated serum and cytokines released by peripheral blood cells after the "in vitro" stimulation with PMA at 1, 12, 24, 36, 48, 56, and 72 hours after the challenge (Table 6). The local ethical committee (IRB-MCK) approved this study and an informed consent in written was obtained from all participants. 
TABLE 1: Characteristics of the patients and control subjects.

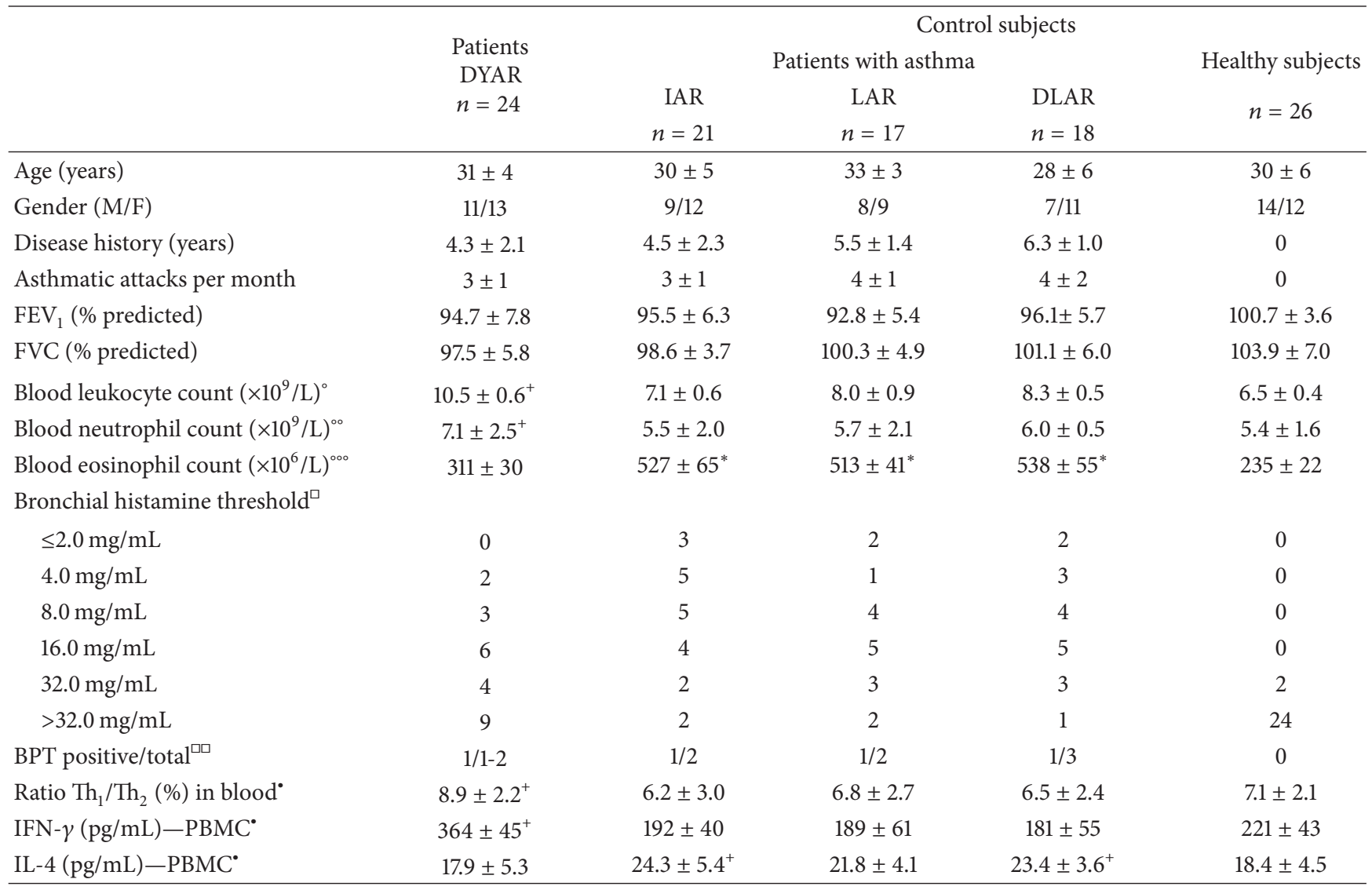

DYAR: delayed asthmatic response; IAR: immediate asthmatic response; LAR: late asthmatic response; DLAR: dual late asthmatic response; asthma: bronchial asthma; values $=$ mean $\pm \mathrm{SD}$; statistical significance as compared with healthy control subjects: ${ }^{*} P<0.05 ;{ }^{+} P \leq 0.05$ (borderline); ${ }^{\circ}$ normal value $=4.0-10 \times$ $10^{9} / \mathrm{L} ;{ }^{\circ}$ normal value $=2.0-7.2 \times 10^{9} / \mathrm{L} ;{ }^{\circ}{ }^{\circ}$ normal value $\leq 300 \times 10^{6} / \mathrm{L} ;{ }^{\square}$ normal value $<32.0 \mathrm{mg} / \mathrm{mL} ;{ }^{\square \square} \mathrm{BPT}$; bronchial provocation tests with allergens (per patient = positive/total); PBMC: peripheral blood mononuclear cells; ${ }^{\circ}$ after stimulation with PMA (Phorbol 12-myristate 13-acetate).

TABLE 2: Survey of the single diagnostic parameters.

Control subjects

Patients Bronchial asthma patients

$$
n=24
$$

IAR LAR DLAR

$n=21$
Healthy subjects

$$
n=26
$$

\begin{tabular}{|c|c|c|c|c|c|}
\hline \multicolumn{6}{|l|}{ Positive skin response (i.c.) } \\
\hline Immediate & 10 & 19 & 5 & 9 & 0 \\
\hline Late & 2 & 2 & 12 & 9 & 0 \\
\hline Delayed & 12 & 0 & 0 & 0 & 0 \\
\hline Increased total $\operatorname{IgE}$ in serum ${ }^{*}$ & 0 & 9 & 4 & 8 & 0 \\
\hline Increased total IgG in serum ${ }^{* * *}$ & 0 & 0 & 10 & 7 & 0 \\
\hline \multicolumn{6}{|l|}{ Increased IgG sub-classes in serum $^{+}$} \\
\hline $\operatorname{IgG}_{1}$ & 0 & 0 & 2 & 1 & 0 \\
\hline $\mathrm{IgG}_{2}$ & 0 & 0 & 0 & 0 & 0 \\
\hline $\operatorname{IgG}_{3}$ & 0 & 0 & 4 & 2 & 1 \\
\hline $\operatorname{IgG}_{4}$ & 0 & 0 & 5 & 2 & 0 \\
\hline Increased total IgM in serum $^{++}$ & 0 & 0 & 0 & 0 & 0 \\
\hline Increased total Ig $\mathrm{A}$ in serum $^{+++}$ & 0 & 0 & 0 & 0 & 0 \\
\hline
\end{tabular}

(i.c.): intracutaneous tests; ${ }^{*}$ total IgE in the serum (PRIST): normal value $=<500 \mathrm{IU} / \mathrm{mL} ;{ }^{* *}$ positive specific IgE in the serum $(\mathrm{RAST})=>0.70 \mathrm{U} / \mathrm{mL}(=\mathrm{more}$ than class 1); ${ }^{* * *}$ total IgG in the serum (single radial immunodiffusion = Mancini technique and ELISA): normal value $=<15.0 \mathrm{~g} / \mathrm{L} ;{ }^{+} \operatorname{IgG}{ }_{1}<5.0 \mathrm{~g} / \mathrm{L} ; \mathrm{IgG}{ }_{2}<$ $2.6 \mathrm{~g} / \mathrm{L} ; \mathrm{IgG}_{3}<0.4 \mathrm{~g} / \mathrm{L} ; \mathrm{IgG}_{4}<0.5 \mathrm{~g} / \mathrm{L} ;{ }^{++} \operatorname{IgM}=<3.8 \mathrm{~g} / \mathrm{L}(<1.5) ;{ }^{+++} \operatorname{IgA}=<4.0 \mathrm{~g} / \mathrm{L}(<3.2)$. 
TABLE 3: Allergens caused particular types of asthmatic response.

\begin{tabular}{|c|c|c|c|c|c|c|}
\hline Allergen & $\begin{array}{c}\text { Concentration } \\
\mathrm{BU} / \mathrm{mL}\end{array}$ & $\begin{array}{l}\text { DYAR } \\
n=24\end{array}$ & $\begin{array}{c}\text { IAR } \\
n=21\end{array}$ & $\begin{array}{c}\text { LAR } \\
n=17\end{array}$ & $\begin{array}{l}\text { DLAR } \\
n=18\end{array}$ & $\begin{array}{c}\text { Healthy subjects } \\
n=26\end{array}$ \\
\hline Dermatophagoides pteronyssinus & 1000 & 5 & 7 & 4 & 6 & 0 \\
\hline Dermatophagoides farinae & 1000 & 1 & 0 & 2 & 1 & 0 \\
\hline \multicolumn{7}{|l|}{ Animal danders } \\
\hline Dog & 3000 & 2 & 3 & 2 & 1 & 0 \\
\hline Cat & 1000 & 2 & 1 & 1 & 2 & 0 \\
\hline Horse & 2000 & 0 & 0 & 1 & 0 & 0 \\
\hline Hamster & 2000 & 0 & 1 & 0 & 0 & 0 \\
\hline Aspergillus fumigatus & 1000 & 1 & 0 & 0 & 1 & 0 \\
\hline \multicolumn{7}{|l|}{ Pollen } \\
\hline Grass mix I & 1000 & 5 & 4 & 3 & 2 & 0 \\
\hline Grass mix II & 1000 & 4 & 2 & 2 & 0 & 0 \\
\hline Tree mix & 3000 & 1 & 1 & 0 & 1 & 0 \\
\hline Weed mix & 1000 & 1 & 0 & 0 & 2 & 0 \\
\hline Birch & 1000 & 1 & 1 & 1 & 1 & 0 \\
\hline Poplar & 2000 & 1 & 0 & 0 & 1 & 0 \\
\hline Ragweed giant & 1000 & 0 & 1 & 1 & 0 & 0 \\
\hline
\end{tabular}

$\mathrm{BU} / \mathrm{mL}$ : biologic units per $1 \mathrm{~mL}$

Grass pollen mix I: Dactylis glomerata, Lolium perenne, Phleum pratensis, and Poa pratensis.

Grass pollen mix II: Festuca pratensis, Holcus lanatus, Agrostis alba, and Anthoxanthum odoratum.

Tree pollen mix: Betula pendula, Corylus avellana, Juniperus communis, and Salix alba.

Weed pollen mix: Artemisia vulgaris, Plantago lanceolata, Rumex acetosa, and Taraxacum officinale.

TABLE 4: Single determination of cytokine concentrations in the (nonstimulated) serum $(\mathrm{pg} / \mathrm{mL})$.

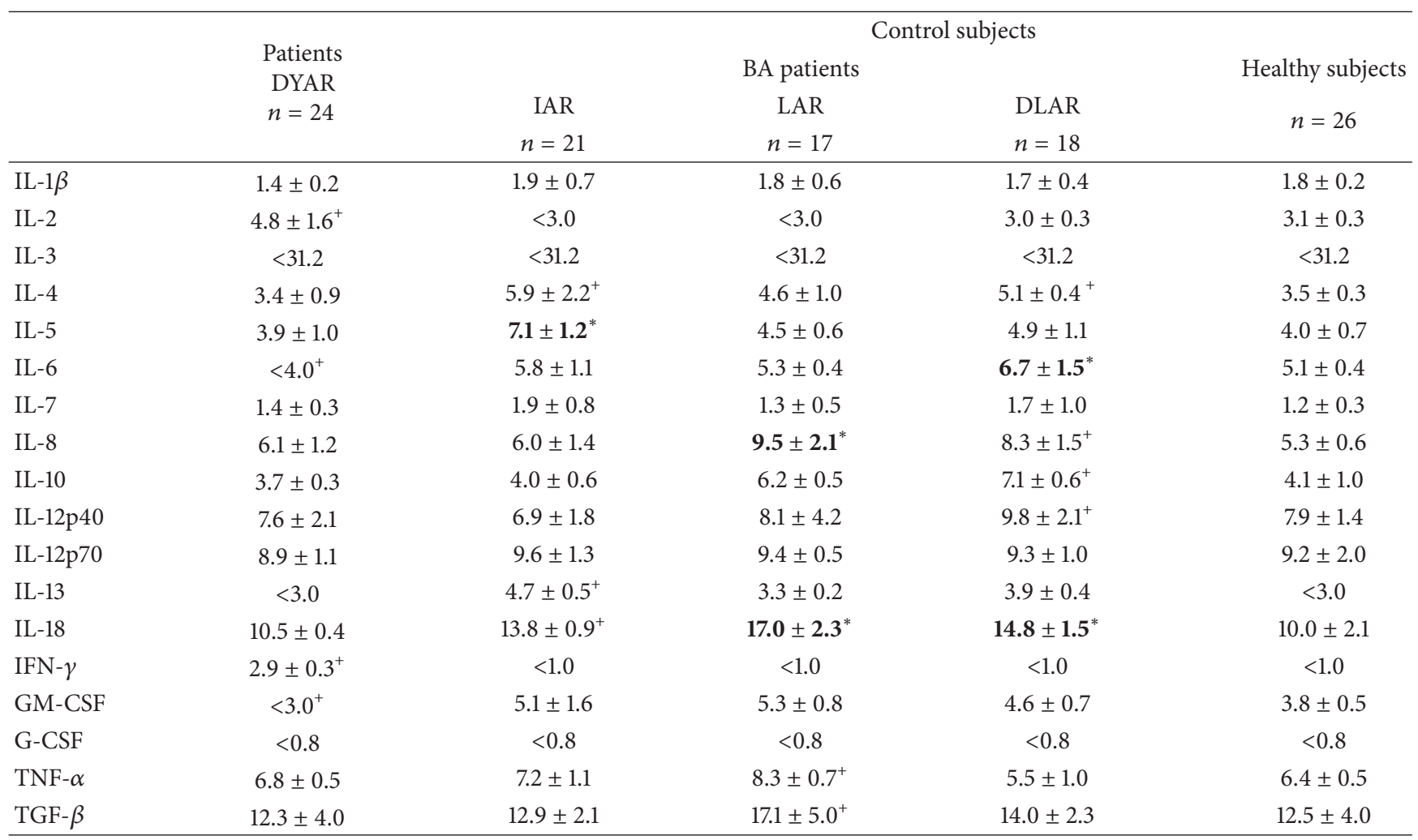

Values $=$ mean \pm SEM; Statistical significance as compared with healthy subject values: ${ }^{*} P<0.05 ;{ }^{+} P \leq 0.05$ (borderline).

DYAR: delayed asthmatic response; IAR: immediate asthmatic response; LAR: late asthmatic response; DLAR: dual late asthmatic response. 
TABLE 5: Mean values of cytokine concentrations in the supernatants $(\mathrm{pg} / \mathrm{mL})$ released from peripheral blood cells stimulated "in vitro" with various nonspecific stimulating agents.

\begin{tabular}{|c|c|c|c|c|c|}
\hline & & & Cont & jects & \\
\hline & DYAR, $n=24$ & & Asthmatics & & Healthy subjects \\
\hline & PHA/PMA/fMLP & $\mathrm{IAR}, n=21$ & LAR, $n=17$ & DLAR, $n=18$ & $n=26$ \\
\hline & & PHA/PMA/fMLP & PHA/PMA/fMLP & PHA/PMA/fMLP & PHA/PMA/fMLP \\
\hline IL-1 $\beta$ & $7.1 / 7.4 / 6.8$ & $7.5 / 7.3 / 7.0$ & $7.4 / 7.7 / 7.5$ & $8.2 / 8.1 / 8.4$ & $8.5 / 8.3 / 8.0$ \\
\hline IL-2 & 4.2/3.9/4.0 & $<3.0$ & $<3.0 / 3.2 /<3.0$ & $<3.0$ & $<3.0$ \\
\hline IL-3 & $<31.2$ & $<31.2$ & $33.2 / 33.5 /<31.2^{+}$ & $<31.2$ & $<31.2$ \\
\hline IL-4 & $19.8 / 21.2 / 20.0$ & $33.8 / 35.9 / 34.7^{*}$ & $24.0 / 23.5 / 23.9$ & $31.0 / 32.8 / 33.0^{*}$ & $21.6 / 22.9 / 21.8$ \\
\hline IL-5 & $5.1 / 6.0 / 5.7$ & $8.7 / 9.5 / 9.3^{*}$ & 9.4/10.6/9.8 ${ }^{*}$ & 9.5/11.0/9.7 ${ }^{*}$ & $4.9 / 5.0 / 4.3$ \\
\hline IL-6 & $4.6 / 5.0 / 5.2$ & 6.4/7.1/7.2* & $4.7 / 5.2 / 5.0$ & 7.3/7.2/8.0 & $4.0 / 4.8 / 3.7$ \\
\hline IL-7 & $3.3 / 4.2 / 4.1$ & $8.9 / 10.0 / 9.4^{*}$ & $3.5 / 3.5 / 3.8$ & $4.1 / 4.4 / 3.4$ & $3.9 / 3.5 / 3.6$ \\
\hline IL-8 & $9.6 / 10.3 / 9.0^{+}$ & 7.4/7.1/7.9 & $10.5 / 11.0 / 10.6^{*}$ & $9.5 / 12.1 / 11.3^{*}$ & $8.2 / 7.0 / 6.5$ \\
\hline IL-10 & $8.1 / 9.0 / 8.5$ & $12.6 / 12.2 / 12.7^{*}$ & $8.3 / 7.6 / 7.9$ & 8.1/8.0/7.9 & $7.7 / 8.4 / 8.2$ \\
\hline IL-12p40 & $9.8 / 10.5 / 10.5$ & $13.9 / 14.4 / 14.2^{+}$ & $15.6 / 15.0 / 15.3^{*}$ & $14.2 / 16.7 / 16.5^{*}$ & $10.1 / 9.6 / 9.5$ \\
\hline IL-12p70 & $13.0 / 14.9 / 13.2^{+}$ & $14.0 / 14.8 / 14.6^{+}$ & $10.3 / 9.9 / 10.1$ & $12.0 / 12.6 / 11.7$ & 11.5/11.9/11.8 \\
\hline IL-13 & $3.8 /<3.0 /<3.0$ & $10.1 / 10.7 / 9.8^{*}$ & $9.9 / 10.3 / 10.0^{*}$ & $11.0 / 11.5 / 10.8^{*}$ & $3.4 / 4.0 /<3.0$ \\
\hline IL-18 & $19.6 / 20.0 / 20.0^{*}$ & $14.1 / 13.9 / 13.7$ & $15.2 / 15.5 / 15.0$ & $16.1 / 15.7 / 15.5$ & $15.9 / 16.5 / 14.2$ \\
\hline IFN- $\gamma$ & $373 / 395 / 350^{*}$ & $199 / 207 / 183$ & $191 / 212 / 205$ & $213 / 218 / 211$ & $224 / 237 / 255$ \\
\hline GM-CSF & $10.4 / 10.5 / 9.6$ & $12.7 / 14.0 / 13.9^{*}$ & $13.5 / 14.3 / 13.8^{*}$ & $15.4 / 15.0 / 14.6^{*}$ & $10.0 / 9.4 / 7.0$ \\
\hline G-CSF & $7.0 / 7.4 / 6.8^{*}$ & $3.9 / 4.5 / 4.5$ & $3.7 / 3.5 / 3.1$ & $4.0 / 4.4 / 3.6$ & $4.1 / 3.5 / 3.2$ \\
\hline TNF- $\alpha$ & $11.7 / 12.9 / 11.3^{+}$ & $8.5 / 8.9 / 9.1$ & $10.4 / 11.7 / 11.6^{+}$ & 7.8/7.5/7.6 & $9.0 / 8.8 / 7.9$ \\
\hline TGF- $\beta$ & $16.8 / 17.5 / 17.4$ & $17.5 / 17.1 / 18.0$ & $20.4 / 22.6 / 21.9^{+}$ & $23.0 / 23.7 / 22.8^{*}$ & $18.0 / 18.2 / 16.7$ \\
\hline
\end{tabular}

DYAR: delayed asthmatic response; IAR: immediate asthmatic response; LAR: late asthmatic response; DLAR: dual late asthmatic response. Values = means \pm SEM. Statistical significance as compared with healthy subject values: ${ }^{*} P<0.05 ;{ }^{+} P \leq 0.05$ (borderline). PHA: Phytohemagglutin in (10 $\mu$ g/mL); PMA: Phorbol 12-myristate 13-acetate $(50 \mathrm{ng} / \mathrm{mL})$; fMLP: $\mathrm{N}$-formylmethionyl-leucyl-phenylalanine $(1 \mu \mathrm{mol} / \mathrm{L})$.

2.2. Control Subjects. The 21 asthmatics demonstrating an isolated IAR, 17 asthmatics developing an isolated LAR, 18 asthmatics showing DLAR, and 26 healthy subjects volunteered to participate as control subjects in this study (Tables $1-5)$.

2.3. Allergens. Dialyzed and lyophilized allergen extracts (Allergopharma, Reinbek, Germany) diluted in PBS were used in concentrations of $100-500 \mathrm{BU} / \mathrm{mL}$ for skin tests and 1000-3000 BU/mL for BPTs (Table 3). The concentrations recommended by the manufacturer were $500 \mathrm{BU} / \mathrm{mL}$ for skin tests and $5000 \mathrm{BU} / \mathrm{mL}$ for the BPTs [40].

2.4. Skin Tests. Scratch tests with allergenic extracts in concentrations of $500 \mathrm{BU} / \mathrm{mL}$ were evaluated after 20 minutes. If they were negative, intracutaneous tests in concentration of $100 \mathrm{BU} / \mathrm{mL}$ and then $500 \mathrm{BU} / \mathrm{mL}$ were performed and evaluated 20 minutes, $6,12,24,36,48,72$, and 96 hours after the intradermal injection. A skin wheal reaction $(>7.0 \mathrm{~mm}$ in diameter) occurring after 20 minutes was qualified as positive immediate skin response, skin infiltration appearing between 6 and 12 hours as a late skin response and skin induration observed later than 48 hours was considered a delayed skin response [40].

2.5. Bronchial Provocation Tests (BPT). The BPTs were performed by means of spirometry (Spirograph D-75, Lode, Groningen, The Netherlands) recording the FVC and $\mathrm{FEV}_{1}$ values. The allergen extracts and PBS were inhaled in the form of an aerosol using the Wiesbadener Doppel-Inhalator at an airflow of $10 \mathrm{~L} / \mathrm{min}$. The nebulizer output was 0.12 $0.14 \mathrm{~mL} / \mathrm{min}$ and the aerosol particles were of a median mass diameter of $2.8-3.6 \mu$.

The BPTs, being a modification of the European standard [42], were performed according to the following schedule: (1) initial (baseline) values recorded at 0,5 , and 10 minutes; (2) PBS control values recorded at 0,5 , and 10 minutes after a 10 minute PBS inhalation; (3) inhalation of allergen aerosol for $2 \times 5$ minutes, with inserted spirometric value measurement, followed by the recording of the $\mathrm{FEV}_{1}$ and $\mathrm{FVC}$ values at $0,5,10,20,30,45,60,90$, and 120 minutes and the every hour up to 12th hour and every second hour during the 22nd and 38th and the 46th and 56th hour intervals. The control challenge with PBS was performed using the same schedule as that applied to the BPTs with allergens. A 5-day interval has always been inserted between the consecutive tests [40].

2.6. Supplementary Parameters. $\mathrm{Th}_{1} / \mathrm{Th}_{2}$ ratio (\%) values in peripheral blood were determined by flow-cytometry and the intracellular IFN- $\gamma$ and IL- 4 in the peripheral blood mononuclear cells (PBMCs) stimulated with PMA were estimated by immunoassay ELISA-kits, as described previously [41].

2.7. Determination of Cytokines. Two series of venous blood were collected in all cases. Samples of $3 \mathrm{~mL}$ blood were, after 
TABLE 6: Nonstimulated plasma concentrations and concentrations of cytokines in supernatants released by cells stimulated "in vitro" with PMA (mean \pm SEM), during DYARs and PBS control tests.

\begin{tabular}{|c|c|c|c|c|c|c|c|c|}
\hline \multirow{2}{*}{ Patients, $n=24$} & \multirow{2}{*}{ Before the challenge } & \multicolumn{7}{|c|}{ After the challenge (hrs) } \\
\hline & & 1 & 12 & 24 & 36 & 48 & 56 & 72 \\
\hline \multicolumn{9}{|l|}{ IL-1 $\beta(\mathrm{pg} / \mathrm{mL})$} \\
\hline \multicolumn{9}{|l|}{ DYAR } \\
\hline $\mathrm{p}$ & $1.6 \pm 0.5$ & $1.5 \pm 0.4$ & $2.0 \pm 1.1$ & $3.3 \pm 1.0$ & $5.9 \pm 0.3^{+}$ & $6.0 \pm 0.7^{+}$ & $3.1 \pm 0.4$ & $2.7 \pm 0.6$ \\
\hline c & $7.3 \pm 1.1$ & $8.4 \pm 0.5$ & $9.6 \pm 1.0$ & $9.2 \pm 0.7$ & $8.8 \pm 0.6$ & $9.3 \pm 0.2$ & $9.0 \pm 1.0$ & $8.6 \pm 0.7$ \\
\hline \multicolumn{9}{|l|}{ PBS } \\
\hline $\mathrm{p}$ & $1.5 \pm 0.2$ & $2.0 \pm 0.7$ & $3.0 \pm 0.5$ & $2.4 \pm 0.3$ & $2.2 \pm 0.6$ & $2.4 \pm 0.8$ & $2.5 \pm 0.7$ & $1.9 \pm 0.4$ \\
\hline c & $7.4 \pm 0.8$ & $7.1 \pm 0.2$ & $8.2 \pm 0.8$ & $8.9 \pm 0.4$ & $9.4 \pm 1.1$ & $9.0 \pm 0.6$ & $8.5 \pm 0.2$ & $8.3 \pm 0.5$ \\
\hline \multicolumn{9}{|l|}{ IL-2 (pg/mL) } \\
\hline \multicolumn{9}{|l|}{ DYAR } \\
\hline $\mathrm{p}$ & $3.5 \pm 0.5$ & $3.5 \pm 0.3$ & $5.2 \pm 0.7^{+}$ & $7.8 \pm 0.6^{*}$ & $7.5 \pm 1.1^{*}$ & $6.9 \pm 0.4^{*}$ & $4.3 \pm 0.5$ & $3.6 \pm 0.3$ \\
\hline c & $3.7 \pm 0.4$ & $5.2 \pm 0.1$ & $8.6 \pm 1.0^{*}$ & $9.3 \pm 0.1^{*}$ & $8.0 \pm 0.7^{*}$ & $9.4 \pm 0.6^{*}$ & $6.7 \pm 0.2^{*}$ & $3.8 \pm 0.5$ \\
\hline \multicolumn{9}{|l|}{ PBS } \\
\hline $\mathrm{p}$ & $3.3 \pm 0.2$ & $3.6 \pm 0.5$ & $3.4 \pm 0.3$ & $<3.0$ & $3.9 \pm 0.5$ & $4.0 \pm 0.7$ & $<3.0$ & $<3.0$ \\
\hline $\mathrm{c}$ & $4.0 \pm 0.8$ & $5.0 \pm 0.9$ & $7.1 \pm 0.4$ & $6.0 \pm 0.3$ & $4.8 \pm 0.6$ & $5.3 \pm 0.2$ & $4.9 \pm 0.4$ & $4.5 \pm 0.4$ \\
\hline \multicolumn{9}{|l|}{ IL-3 (pg/mL) } \\
\hline \multicolumn{9}{|l|}{ DYAR } \\
\hline $\mathrm{p}$ & $<31.2$ & $<31.2$ & $<31.2$ & $<31.2$ & $32.2 \pm 0.5$ & $31.7 \pm 0.6$ & $32.0 \pm 0.4$ & $<31.2$ \\
\hline c & $34.2 \pm 2.0$ & $36.7 \pm 2.4$ & $38.0 \pm 3.9$ & $35.5 \pm 1.2$ & $37.6 \pm 2.7$ & $35.1 \pm 1.5$ & $36.9 \pm 1.8$ & $34.7 \pm 1.0$ \\
\hline \multicolumn{9}{|l|}{ PBS } \\
\hline $\mathrm{p}$ & $<31.2$ & $31.2 \pm 0.0$ & $<31.2$ & $31.5 \pm 0.2$ & $<31.2$ & $<31.2$ & $31.3 \pm 0.1$ & $<31.2$ \\
\hline c & $32.5 \pm 1.8$ & $35.3 \pm 1.8$ & $35.9 \pm 1.2$ & $37.4 \pm 0.9$ & $36.5 \pm 1.4$ & $33.7 \pm 1.3$ & $35.5 \pm 2.0$ & $35.2 \pm 0.6$ \\
\hline \multicolumn{9}{|l|}{ IL-4 (pg/mL) } \\
\hline \multicolumn{9}{|l|}{ DYAR } \\
\hline $\mathrm{p}$ & $4.5 \pm 0.8$ & $4.0 \pm 0.5$ & $5.4 \pm 0.6$ & $5.0 \pm 1.0$ & $4.6 \pm 0.4$ & $5.5 \pm 1.2$ & $4.8 \pm 0.5$ & $4.2 \pm 0.4$ \\
\hline c & $23.7 \pm 1.6$ & $25.0 \pm 2.7$ & $24.3 \pm 1.9$ & $22.0 \pm 2.3$ & $23.6 \pm 0.5$ & $22.9 \pm 0.8$ & $23.4 \pm 1.1$ & $23.1 \pm 0.5$ \\
\hline \multicolumn{9}{|l|}{ PBS } \\
\hline $\mathrm{p}$ & $3.9 \pm 0.5$ & $3.8 \pm 0.6$ & $4.2 \pm 1.1$ & $4.5 \pm 0.6$ & $5.4 \pm 0.3$ & $3.9 \pm 0.6$ & $4.0 \pm 1.0$ & $4.1 \pm 0.5$ \\
\hline c & $22.9 \pm 1.1$ & $21.7 \pm 1.0$ & $22.6 \pm 0.8$ & $23.4 \pm 1.5$ & $22.7 \pm 1.6$ & $22.2 \pm 1.0$ & $21.6 \pm 0.9$ & $22.5 \pm 1.1$ \\
\hline \multicolumn{9}{|l|}{ IL-5 (pg/mL) } \\
\hline \multicolumn{9}{|l|}{ DYAR } \\
\hline $\mathrm{p}$ & $4.1 \pm 0.8$ & $4.9 \pm 0.9$ & $4.5 \pm 1.1$ & $4.3 \pm 0.6$ & $4.5 \pm 0.5$ & $5.0 \pm 1.0$ & $4.4 \pm 0.7$ & $4.6 \pm 0.4$ \\
\hline c & $6.9 \pm 1.0$ & $7.7 \pm 0.8$ & $7.5 \pm 1.3$ & $8.0 \pm 0.4$ & $7.1 \pm 0.7$ & $7.3 \pm 2.1$ & $7.6 \pm 1.4$ & $7.4 \pm 0.9$ \\
\hline \multicolumn{9}{|l|}{ PBS } \\
\hline $\mathrm{p}$ & $3.8 \pm 0.5$ & $3.7 \pm 0.6$ & $4.3 \pm 0.5$ & $4.1 \pm 1.5$ & $3.9 \pm 0.4$ & $4.8 \pm 1.0$ & $4.2 \pm 0.9$ & $4.5 \pm 0.6$ \\
\hline c & $6.5 \pm 0.3$ & $7.5 \pm 1.4$ & $8.0 \pm 0.7$ & $7.9 \pm 1.1$ & $7.2 \pm 0.6$ & $7.2 \pm 0.4$ & $7.3 \pm 0.5$ & $7.6 \pm 1.0$ \\
\hline \multicolumn{9}{|l|}{ IL-6 (pg/mL) } \\
\hline DYAR & & & & & & & & \\
\hline $\mathrm{p}$ & $<4.0$ & $<4.0$ & $4.5 \pm 04$ & $<4.0$ & $4.3 \pm 0.2$ & $<4.0$ & $<4.0$ & $<4.0$ \\
\hline c & $5.7 \pm 1.1$ & $7.8 \pm 0.9$ & $8.1 \pm 1.3$ & $8.6 \pm 1.7$ & $8.4 \pm 0.6$ & $7.9 \pm 1.0$ & $7.5 \pm 0.4$ & $7.0 \pm 0.8$ \\
\hline PBS & & & & & & & & \\
\hline $\mathrm{p}$ & $<4.0$ & $<4.0$ & $<4.0$ & $<4.0$ & $4.7 \pm 0.5$ & $<4.0$ & $4.2 \pm 0.7$ & $4.1 \pm 0.3$ \\
\hline c & $5.9 \pm 0.5$ & $6.1 \pm 1.3$ & $7.2 \pm 1.0$ & $7.9 \pm 2.3$ & $8.0 \pm 1.1$ & $7.4 \pm 0.3$ & $7.7 \pm 0.8$ & $7.5 \pm 0.5$ \\
\hline IL-7 (pg/mL) & & & & & & & & \\
\hline DYAR & & & & & & & & \\
\hline $\mathrm{p}$ & $1.7 \pm 0.2$ & $<0.1^{*}$ & $0.7 \pm 0.2$ & $0.3 \pm 0.1^{+}$ & $<0.1^{*}$ & $<0.1^{*}$ & $<0.1^{*}$ & $0.2 \pm 0.1^{+}$ \\
\hline c & $4.3 \pm 0.5$ & $5.7 \pm 1.0$ & $6.2 \pm 0.8$ & $6.5 \pm 0.4$ & $5.9 \pm 1.0$ & $6.6 \pm 0.7$ & $6.3 \pm 0.4$ & $5.8 \pm 0.3$ \\
\hline PBS & & & & & & & & \\
\hline $\mathrm{p}$ & $2.0 \pm 0.1$ & $2.1 \pm 0.8$ & $1.7 \pm 0.4$ & $1.3 \pm 0.5$ & $2.5 \pm 0.7$ & $2.1 \pm 0.4$ & $1.7 \pm 0.9$ & $2.2 \pm 1.1$ \\
\hline c & $4.6 \pm 0.3$ & $4.9 \pm 0.5$ & $5.5 \pm 1.0$ & $5.8 \pm 0.9$ & $6.2 \pm 1.3$ & $6.3 \pm 1.1$ & $5.6 \pm 0.7$ & $5.3 \pm 0.6$ \\
\hline
\end{tabular}


TABle 6: Continued.

\begin{tabular}{|c|c|c|c|c|c|c|c|c|}
\hline \multirow{2}{*}{ Patients, $n=24$} & \multirow{2}{*}{ Before the challenge } & \multicolumn{7}{|c|}{ After the challenge (hrs) } \\
\hline & & 1 & 12 & 24 & 36 & 48 & 56 & 72 \\
\hline \multicolumn{9}{|l|}{ IL-8 (pg/mL) } \\
\hline \multicolumn{9}{|l|}{ DYAR } \\
\hline $\mathrm{p}$ & $6.8 \pm 1.0$ & $7.0 \pm 1.1$ & $7.2 \pm 0.5$ & $6.7 \pm 0.8$ & $6.5 \pm 0.4$ & $11.5 \pm 0.9^{*}$ & $11.8 \pm 1.0^{*}$ & $7.0 \pm 0.5$ \\
\hline c & $12.2 \pm 2.1$ & $12.6 \pm 1.8$ & $13.5 \pm 2.0$ & $16.9 \pm 2.6^{*}$ & $17.6 \pm 1.7^{*}$ & $16.8 \pm 2.0^{*}$ & $12.9 \pm 1.8$ & $12.8 \pm 1.4$ \\
\hline \multicolumn{9}{|l|}{ PBS } \\
\hline $\mathrm{p}$ & $7.2 \pm 1.8$ & $7.5 \pm 2.0$ & $6.8 \pm 1.2$ & $7.3 \pm 0.5$ & $7.1 \pm 0.6$ & $6.9 \pm 0.8$ & $7.3 \pm 0.5$ & $7.0 \pm 0.7$ \\
\hline c & $11.4 \pm 0.6$ & $12.5 \pm 1.0$ & $12.9 \pm 1.5$ & $13.6 \pm 1.8$ & $14.0 \pm 1.9$ & $13.5 \pm 1.1$ & $13.5 \pm 2.1$ & $13.2 \pm 1.0$ \\
\hline \multicolumn{9}{|l|}{ IL-10 (pg/mL) } \\
\hline \multicolumn{9}{|l|}{ DYAR } \\
\hline $\mathrm{p}$ & $3.5 \pm 0.4$ & $3.6 \pm 0.4$ & $3.8 \pm 1.0$ & $5.5 \pm 0.4^{+}$ & $4.1 \pm 1.0$ & $3.4 \pm 0.7$ & $3.8 \pm 0.9$ & $4.1 \pm 0.6$ \\
\hline c & $10.9 \pm 2.2$ & $10.3 \pm 0.6$ & $11.5 \pm 0.8$ & $11.2 \pm 0.3$ & $12.4 \pm 1.3$ & $11.7 \pm 0.5$ & $11.0 \pm 1.0$ & $11.3 \pm 0.4$ \\
\hline \multicolumn{9}{|l|}{ PBS } \\
\hline $\mathrm{p}$ & $<3.0$ & $<3.0$ & $<3.0$ & $<3.0$ & $<3.0$ & $<3.0$ & $3.2 \pm 0.1$ & $<3.0$ \\
\hline $\mathrm{c}$ & $9.5 \pm 0.6$ & $10.0 \pm 0.3$ & $9.8 \pm 0.6$ & $10.9 \pm 1.1$ & $11.2 \pm 0.7$ & $10.8 \pm 0.4$ & $9.9 \pm 0.3$ & $10.2 \pm 0.5$ \\
\hline \multicolumn{9}{|l|}{ IL-12p40 (pg/mL) } \\
\hline \multicolumn{9}{|l|}{ DYAR } \\
\hline $\mathrm{p}$ & $8.0 \pm 2.0$ & $9.0 \pm 3.2$ & $8.1 \pm 1.1$ & $8.8 \pm 1.9$ & $8.2 \pm 0.6$ & $7.8 \pm 0.7$ & $8.5 \pm 0.4$ & $8.1 \pm 1.0$ \\
\hline c & $10.7 \pm 0.6$ & $11.8 \pm 1.3$ & $12.5 \pm 2.2$ & $11.6 \pm 0.8$ & $10.5 \pm 1.0$ & $11.4 \pm 0.9$ & $11.0 \pm 1.1$ & $11.3 \pm 0.6$ \\
\hline \multicolumn{9}{|l|}{ PBS } \\
\hline $\mathrm{p}$ & $7.7 \pm 1.3$ & $7.9 \pm 0.4$ & $7.6 \pm 0.5$ & $7.9 \pm 1.3$ & $7.5 \pm 1.0$ & $8.0 \pm 0.6$ & $7.4 \pm 0.9$ & $7.9 \pm 0.8$ \\
\hline c & $11.0 \pm 1.4$ & $12.1 \pm 2.5$ & $12.8 \pm 2.0$ & $11.9 \pm 0.7$ & $12.3 \pm 2.5$ & $11.6 \pm 0.4$ & $11.9 \pm 1.8$ & $11.7 \pm 1.2$ \\
\hline \multicolumn{9}{|l|}{ IL-12p70 (pg/mL) } \\
\hline \multicolumn{9}{|l|}{ DYAR } \\
\hline $\mathrm{p}$ & $9.0 \pm 1.9$ & $10.3 \pm 2.0$ & $11.5 \pm 2.1$ & $19.3 \pm 3.2^{*}$ & $17.9 \pm 3.0^{*}$ & $16.8 \pm 1.3^{*}$ & $9.9 \pm 1.0$ & $10.2 \pm 0.5$ \\
\hline c & $15.5 \pm 2.8$ & $15.4 \pm 1.9$ & $18.2 \pm 3.0$ & $24.5 \pm 2.4^{*}$ & $25.8 \pm 1.6^{*}$ & $27.9 \pm 3.5^{*}$ & $27.2 \pm 2.8^{*}$ & $16.9 \pm 1.4$ \\
\hline \multicolumn{9}{|l|}{ PBS } \\
\hline $\mathrm{p}$ & $10.4 \pm 2.1$ & $10.1 \pm 1.0$ & $10.9 \pm 1.2$ & $10.0 \pm 1.3$ & $9.8 \pm 0.8$ & $9.9 \pm 1.0$ & $10.1 \pm 0.7$ & $9.6 \pm 1.2$ \\
\hline $\mathrm{c}$ & $16.0 \pm 3.3$ & $16.2 \pm 0.8$ & $16.9 \pm 1.3$ & $18.0 \pm 0.5$ & $17.7 \pm 1.4$ & $18.1 \pm 0.6$ & $18.5 \pm 1.1$ & $17.7 \pm 1.5$ \\
\hline \multicolumn{9}{|l|}{ IL-13 (pg/mL) } \\
\hline \multicolumn{9}{|l|}{ DYAR } \\
\hline $\mathrm{p}$ & $<3.0$ & $<3.0$ & $4.1 \pm 0.5$ & $<3.0$ & $3.7 \pm 0.4$ & $6.9 \pm 0.7^{*}$ & $8.2 \pm 0.9^{*}$ & $7.3 \pm 1.0^{*}$ \\
\hline c & $6.6 \pm 0.3$ & $7.1 \pm 1.0$ & $8.4 \pm 0.7^{+}$ & $8.1 \pm 0.9$ & $9.8 \pm 1.1^{*}$ & $10.2 \pm 1.3^{*}$ & $8.5 \pm 1.1^{+}$ & $6.9 \pm 0.5$ \\
\hline \multicolumn{9}{|l|}{ PBS } \\
\hline $\mathrm{p}$ & $<3.0$ & $<3.0$ & $<3.0$ & $<3.0$ & $<3.0$ & $<3.0$ & $3.1 \pm 0.1$ & $<3.0$ \\
\hline $\mathrm{c}$ & $7.0 \pm 0.8$ & $7.5 \pm 1.2$ & $7.1 \pm 0.9$ & $8.3 \pm 1.5$ & $8.6 \pm 2.0$ & $8.1 \pm 1.4$ & $7.9 \pm 0.5$ & $7.6 \pm 0.8$ \\
\hline \multicolumn{9}{|l|}{ IL-18 (pg/mL) } \\
\hline DYAR & & & & & & & & \\
\hline $\mathrm{p}$ & $11.0 \pm 1.7$ & $11.4 \pm 2.3$ & $12.3 \pm 2.0$ & $11.5 \pm 1.0$ & $13.9 \pm 0.5^{+}$ & $14.6 \pm 1.0^{*}$ & $14.8 \pm 0.5^{*}$ & $10.5 \pm 0.8$ \\
\hline c & $20.1 \pm 1.3$ & $19.6 \pm 1.0$ & $23.4 \pm 0.8$ & $26.7 \pm 1.5^{+}$ & $27.5 \pm 1.2^{*}$ & $28.0 \pm 2.6^{*}$ & $30.1 \pm 4.0^{*}$ & $26.3 \pm 2.7$ \\
\hline PBS & & & & & & & & \\
\hline $\mathrm{p}$ & $11.3 \pm 2.9$ & $12.0 \pm 1.5$ & $11.1 \pm 0.9$ & $12.0 \pm 0.2$ & $11.0 \pm 0.6$ & $10.8 \pm 1.0$ & $10.4 \pm 0.7$ & $11.2 \pm 0.4$ \\
\hline c & $19.7 \pm 0.8$ & $20.4 \pm 2.0$ & $22.7 \pm 2.5$ & $21.9 \pm 1.4$ & $22.6 \pm 0.8$ & $22.8 \pm 0.7$ & $21.8 \pm 1.4$ & $20.9 \pm 1.6$ \\
\hline IFN- $\gamma(\mathrm{pg} / \mathrm{mL})$ & & & & & & & & \\
\hline DYAR & & & & & & & & \\
\hline $\mathrm{p}$ & $2.4 \pm 0.3$ & $2.7 \pm 0.7$ & $4.2 \pm 0.6$ & $14.1 \pm 2.0^{*}$ & $17.9 \pm 2.1^{*}$ & $15.7 \pm 1.8^{*}$ & $8.0 \pm 1.0^{*}$ & $2.9 \pm 0.5$ \\
\hline c & $361 \pm 108$ & $383 \pm 115$ & $405 \pm 111$ & $475 \pm 131^{*}$ & $489 \pm 125^{*}$ & $503 \pm 89^{*}$ & $466 \pm 103^{*}$ & $401 \pm 114$ \\
\hline PBS & & & & & & & & \\
\hline $\mathrm{p}$ & $2.1 \pm 0.2$ & $2.9 \pm 0.3$ & $2.3 \pm 1.1$ & $2.4 \pm 0.3$ & $2.2 \pm 0.9$ & $2.1 \pm 0.4$ & $2.3 \pm 0.2$ & $2.5 \pm 0.6$ \\
\hline c & $367 \pm 91$ & $354 \pm 68$ & $288 \pm 94$ & $339 \pm 102$ & $321 \pm 113$ & $344 \pm 116$ & $352 \pm 97$ & $370 \pm 105$ \\
\hline
\end{tabular}


TABLE 6: Continued.

\begin{tabular}{|c|c|c|c|c|c|c|c|c|}
\hline \multirow{2}{*}{ Patients, $n=24$} & \multirow{2}{*}{ Before the challenge } & \multicolumn{7}{|c|}{ After the challenge (hrs) } \\
\hline & & 1 & 12 & 24 & 36 & 48 & 56 & 72 \\
\hline \multicolumn{9}{|c|}{ GM-CSF (pg/mL) } \\
\hline \multicolumn{9}{|l|}{ DYAR } \\
\hline $\mathrm{p}$ & $<3.0$ & $3.5 \pm 0.3$ & $4.2 \pm 0.6^{+}$ & $5.1 \pm 1.3^{*}$ & $4.9 \pm 0.7^{+}$ & $3.1 \pm 0.1$ & $3.0 \pm 0.0$ & $<3.0$ \\
\hline c & $10.2 \pm 0.4$ & $11.8 \pm 0.9$ & $13.5 \pm 1.7$ & $12.8 \pm 1.0$ & $13.1 \pm 2.2$ & $12.5 \pm 0.8$ & $11.4 \pm 1.0$ & $11.2 \pm 0.5$ \\
\hline \multicolumn{9}{|l|}{ PBS } \\
\hline $\mathrm{p}$ & $<3.0$ & $<3.0$ & $<3.0$ & $<3.0$ & $3.0 \pm 0.0$ & $<3.0$ & $<3.0$ & $<3.0$ \\
\hline c & $10.7 \pm 0.6$ & $10.4 \pm 0.5$ & $12.3 \pm 1.0$ & $11.9 \pm 1.2$ & $11.0 \pm 1.4$ & $11.3 \pm 0.6$ & $10.8 \pm 1.2$ & $11.5 \pm 1.0$ \\
\hline \multicolumn{9}{|l|}{ G-CSF (pg/mL) } \\
\hline \multicolumn{9}{|l|}{ DYAR } \\
\hline $\mathrm{p}$ & $<0.8$ & $1.7 \pm 0.4$ & $6.5 \pm 1.8^{*}$ & $6.9 \pm 0.7^{*}$ & $5.4 \pm 0.9^{*}$ & $1.5 \pm 0.4$ & $<0.8$ & $<0.8$ \\
\hline c & $7.1 \pm 0.6$ & $8.4 \pm 1.0$ & $8.9 \pm 0.7^{+}$ & $9.5 \pm 2.2^{+}$ & $9.0 \pm 1.6^{+}$ & $8.6 \pm 0.7$ & $7.5 \pm 1.2$ & $7.8 \pm 1.1$ \\
\hline \multicolumn{9}{|l|}{ PBS } \\
\hline $\mathrm{p}$ & $<0.8$ & $<0.8$ & $<0.8$ & $1.0 \pm 0.1$ & $0.9 \pm 0.1$ & $<0.8$ & $<0.8$ & $<0.8$ \\
\hline c & $7.5 \pm 0.4$ & $7.2 \pm 0.5$ & $7.6 \pm 0.9$ & $8.7 \pm 1.3$ & $8.2 \pm 0.5$ & $8.0 \pm 1.3$ & $7.9 \pm 1.0$ & $7.4 \pm 0.6$ \\
\hline \multicolumn{9}{|l|}{ TNF- $\alpha(\mathrm{pg} / \mathrm{mL})$} \\
\hline \multicolumn{9}{|l|}{ DYAR } \\
\hline $\mathrm{p}$ & $7.1 \pm 2.0$ & $8.0 \pm 1.7$ & $7.4 \pm 0.8$ & $8.2 \pm 1.2$ & $14.5 \pm 2.0^{*}$ & $12.4 \pm 1.6^{*}$ & $13.0 \pm 0.5^{*}$ & $8.0 \pm 0.7$ \\
\hline c & $12.9 \pm 1.3$ & $13.4 \pm 0.8$ & $16.6 \pm 1.1^{+}$ & $17.3 \pm 0.5^{*}$ & $17.9 \pm 0.7^{*}$ & $18.0 \pm 2.2^{*}$ & $17.5 \pm 1.0^{*}$ & $15.4 \pm 1.1$ \\
\hline \multicolumn{9}{|l|}{ PBS } \\
\hline $\mathrm{p}$ & $6.7 \pm 1.1$ & $7.0 \pm 0.5$ & $6.9 \pm 0.8$ & $6.5 \pm 0.3$ & $7.4 \pm 1.4$ & $7.0 \pm 1.0$ & $7.2 \pm 0.8$ & $6.5 \pm 0.3$ \\
\hline $\mathrm{c}$ & $13.2 \pm 0.8$ & $13.6 \pm 1.0$ & $14.1 \pm 2.0$ & $14.7 \pm 1.8$ & $15.0 \pm 1.6$ & $14.9 \pm 2.1$ & $15.1 \pm 1.3$ & $14.6 \pm 0.5$ \\
\hline \multicolumn{9}{|l|}{ TGF- $\beta$ (pg/mL) } \\
\hline \multicolumn{9}{|l|}{ DYAR } \\
\hline $\mathrm{p}$ & $11.0 \pm 2.3$ & $12.5 \pm 2.3$ & $24.5 \pm 3.7^{*}$ & $21.1 \pm 2.8^{*}$ & $19.0 \pm 2.5^{*}$ & $18.4 \pm 1.0^{*}$ & $11.3 \pm 0.5$ & $10.8 \pm 0.3$ \\
\hline c & $17.2 \pm 1.7$ & $21.4 \pm 3.1$ & $29.5 \pm 2.2^{*}$ & $34.3 \pm 2.0^{*}$ & $30.1 \pm 1.9^{*}$ & $24.8 \pm 2.5^{+}$ & $18.7 \pm 1.5$ & $18.2 \pm 0.9$ \\
\hline \multicolumn{9}{|l|}{ PBS } \\
\hline $\mathrm{p}$ & $10.1 \pm 1.2$ & $10.5 \pm 0.8$ & $11.3 \pm 0.2$ & $10.8 \pm 1.0$ & $10.4 \pm 0.7$ & $11.0 \pm 0.5$ & $10.6 \pm 0.7$ & $11.5 \pm 0.4$ \\
\hline c & $18.0 \pm 1.1$ & $18.8 \pm 1.0$ & $20.9 \pm 0.8$ & $19.4 \pm 1.3$ & $21.2 \pm 1.0$ & $20.5 \pm 0.9$ & $19.0 \pm 1.0$ & $19.3 \pm 0.6$ \\
\hline
\end{tabular}

DYAR: delayed asthmatic response; PBS: phosphate buffered saline (control); PMA: phorbol 12-myristate 12-acetate.

Values of cytokines $=$ means \pm SEM; statistical significance of the cytokine concentrations as compared with their prechallenge $($ baseline $)$ values: ${ }^{*} P<0.05$, ${ }^{+} P \leq 0.05$ (borderline); p: plasma; c: supernatants of cells stimulated with PMA.

clotting, centrifuged at $3000 \times \mathrm{g}$ for 10 minutes and serum aliquots were stored at $-70^{\circ} \mathrm{C}$. Samples of $6 \mathrm{~mL}$ heparinized blood were centrifuged at $1800 \mathrm{~g}$ for 5 minutes at $4^{\circ} \mathrm{C}$ and plasma aliquots were stored at $-70^{\circ} \mathrm{C}$. The unseparated blood cells were washed in RPMI 1640 (Sigma-Aldrich, St. Louis, USA), centrifuged at $2000 \times \mathrm{g}$ for 10 minutes at $4^{\circ} \mathrm{C}$ and resuspended in RPMI 1640 supplemented with penicillin $(100 \mathrm{IU} / \mathrm{mL})$, streptomycin $(100 \mu \mathrm{g} / \mathrm{mL})$, and L-glutamine $(2 \mathrm{mmol} / \mathrm{L})$ at a concentration of $5 \times 10^{6}$ leukocytes $/ \mathrm{mL}$. The cell viability was confirmed by trypan blue dye exclusion. The cell suspension was divided into 4 equal portions. Three portions, stimulated with $10 \mu \mathrm{g} / \mathrm{mL}$ of PHA (Wellcome Diagnostics, Dartford, UK), PMA (Sigma-Aldrich) in a concentration of $50 \mathrm{ng} / \mathrm{mL}$ and $1 \mu \mathrm{g} / \mathrm{mL}$ ionomycin or $1 \mu \mathrm{mol} / \mathrm{L}$ of fMLP (Sigma-Aldrich), and the nonstimulated control portion, were cultured for 24 hours at $37^{\circ} \mathrm{C}$ under $5 \% \mathrm{CO}_{2}$ in a humidified incubator. The supernatants were collected by centrifugation at $2000 \times \mathrm{g}$ for 15 minutes and aliquots were stored at $-70^{\circ} \mathrm{C}$. The cytokines in the supernatants as well as in the nonstimulated plasma were estimated by commercial immunoassay (ELISA, EIA) kits, following the manufacturers' recommendations. All measurements were performed in duplicate. The detection limits in $\mathrm{pg} / \mathrm{mL}$ are reported in brackets. The cytokines Il-1 $\beta$ (1.0), IL-2 $(<3.0)$, IL-3 (31.2), IL-5 (3.0), IL-6 (4.0), IL-7 (0.1), IL-10 (<3.0), IL-13 (<3.0), GM-CSF (<3.0), G-CSF (0.8), TNF- $\alpha(6.0)$, and TGF- $\beta$ (6.0) were measured using the R \& D System (Minneapolis/MN, USA) kits, IL-4 (0.6), IL-8 (1.3), IL-12p70 (2.1), IL-18 (9.2), and IFN- $\gamma$ (1.0) by Bender MedSystems (Wien, Austria), and IL-12p40 (3.9) by Becton Dickinson (San Jose, USA) kits. The interassay as well as intraassay coefficients of variations for these kits were less than $10 \%$.

2.8. Statistical Analysis. The initial and repeated DYAR and PBS controls were statistically evaluated by means of fitting polynomials to the mean curves over time; eight time points within 120 minutes and twenty-four time points up to 56 hours after the challenge. The hypotheses were tested by the generalized multivariate analysis of the variance model (MANOVA) [43]. 
The postchallenge cytokine values recorded at each of the time points during the repeated DYAR and PBS controls in individual patients were compared with their prechallenge values and statistically analyzed by Wilcoxon matched-pair signed rank test. The mean postchallenge values of individual cytokines measured at each of the time points during the repeated DYARs were compared with the corresponding mean PBS values and statistically evaluated by MannWhitney $U$ test. A $P$ value $<0.05$ was considered to be statistically significant.

\section{Results}

3.1. Initial DYAR. The DYAR beginning between 26 and 32 hours, reaching its maximum between 32 and 48 hours and resolving within 56 hours after the allergen challenge (Figure 1) was significantly positive both in comparison of the postchallenge with the prechallenge $\operatorname{FEV}_{1}$ values $(P<$ $0.01)$ and with the PBS values $(P<0.001)$. No significant differences in the appearance of DYAR were found with respect to the individual allergens $(P=0.21)$ (Table 3$)$.

The DYAR was associated with increased blood leukocyte, neutrophil, and lymphocyte counts (Table 1), changed $\mathrm{Th}_{1} / \mathrm{Th}_{2}$ cell ratio in peripheral blood in favour of $\mathrm{Th}_{1}$ cells $(P<0.01)$, increased intracellular concentration of IFN- $\gamma$ $(P<0.05)$ but not of IL-4 $(P>0.05)$ (Table 1$)$, decreased bronchial histamine threshold in 63\% (Table 1), positive immediate skin response in $42 \%$, and positive delayed skin response in $50 \%$ (Table 2 ).

3.2. Repeated DYAR. The repeated DYAR was also significantly positive both comparing the postchallenge values with the prechallenge $\mathrm{FEV}_{1}$ values $(P=0.001)$ and with the corresponding PBS values $(P=0.001)$ (Figure 1$)$. No statistically significant differences were found between the initial and the repeated DYARs $(P=0.17)$.

\subsection{Prechallenge Cytokine Concentrations in Nonspecifically} Stimulated Blood Cells. The prechallenge concentrations of some cytokines in the nonstimulated serum of the DYAR patients, such as IL-2, IL-6, IFN- $\gamma$, and GM-CSF, differed slightly, but not significantly, from those recorded in the healthy subjects as well as from the control asthmatics demonstrated IAR, LAR, and DLAR (Table 4).

The prechallenge cytokine concentrations released by the "in vitro" stimulated peripheral blood cells into supernatants of DYAR patients differed from those measured both in the healthy subjects and in the control asthmatics developing other asthmatic response types (Table 5). The mean cytokine concentrations released after stimulation with PHA, PMA, or fMLP did not differ significantly with respect to the particular agent, although the fMLP values were slightly lower than the PHA or PMA values (Table 5). No significant differences $(P>$ 0.05 ) were found between the concentrations of individual cytokines recorded in the nonstimulated serum (Table 4) and those in the nonstimulated plasma (Table 6) of the DYAR patients.
3.4. Postchallenge Cytokine Concentrations in Nonspecifically Stimulated Blood Cells. The DYAR was associated with significant changes $(P=0.032-0.045)$ of the postchallenge cytokine concentrations, as compared both with their prechallenge values and with the corresponding PBS control values, as follows: (i) In the nonstimulated plasma: increased concentrations of IL-2, IL-8, IL-12p70, IL-13, IL-18, IFN- $\gamma$, GCSF, TNF- $\alpha$, and TGF- $\beta$; a decreased concentration of IL7; (ii) In the supernatants of the blood cells stimulated with PMA: increased concentrations of IL-2, IL-8, IL-12p70, IL-13, IL-18, IFN- $\gamma$, TNF- $\alpha$, and TGF- $\beta$ (Table 6 , Figures 2 (a) and 2(b)). Most of these changes occurred 24-56 hours after the allergen challenge, which is the time of the maximal DYAR performance (Figure 1). No significant changes in the plasma $(P>0.05)$ or supernatants $(P>0.1)$ were found during the PBS controls.

3.5. Control Subjects. In the control patients demonstrating IAR, LAR or DLAR, as compared with healthy control subjects, significant changes in the concentrations of various cytokines both in the nonstimulated serum (Table 4) and in the supernatants of the "in vitro" stimulated blood cells (Table 5) were recorded. These changes differed significantly from those measured in the DYAR patients.

\section{Discussion}

The DYAR differs from the IAR, LAR, and DLAR in clinical course, immunologic features and pharmacologic modulation $[10,11,19,20,40,41]$. The DYAR is associated with profiles of eicosanoids, adhesion molecules, and cytokine in peripheral blood different from those accompanying the IAR, LAR, and DLAR, suggesting involvement of different immunologic mechanisms in the particular asthmatic response types $[1,2$, 5-18, 21-41, 44, 45].

Cytokines, with their manifold biologic effects, represent very important components of the immunologic mechanisms $[1,2,6,9,13,23-39]$. They participate in various stages of the immunologic mechanisms not only as intercellular signal transmitting factors, but also as a regulatory, for example, stimulating, inhibiting, and chemotactic, factors for most cell types involved in immunologic processes $[1,2,6-9,11$, 13-18, 21-39]. Additionally, various cytokines can act by a synergistic or antagonistic manner among them. Their role in the allergic bronchial asthma and their significance for the diagnostic conclusions have already been investigated from various points of view [1, 2, 5-9, 13-18, 21-39].

In most of these studies, a single measurement of various cytokines in the asthma patients was performed $[13,14,17$, $26,32,35,39]$. In some of the studies, the cytokines were determined in the bronchoalveolar lavage (BAL) fluid or (induced) sputum after the segmental allergen challenge [8, $9,15,18,21,22,24,25,27-30,34]$. In only few papers, the cytokines were studied in peripheral blood $(\mathrm{PB})$ during the particular types of asthmatic response, such as IAR, LAR, or DLAR, due to the bronchial challenge with inhaled allergens (BPT) $[18,21,23]$. Moreover, in these infrequent studies, the intracellular cytokines were usually determined in the supernatants of the isolated blood cell after their "in vitro" 


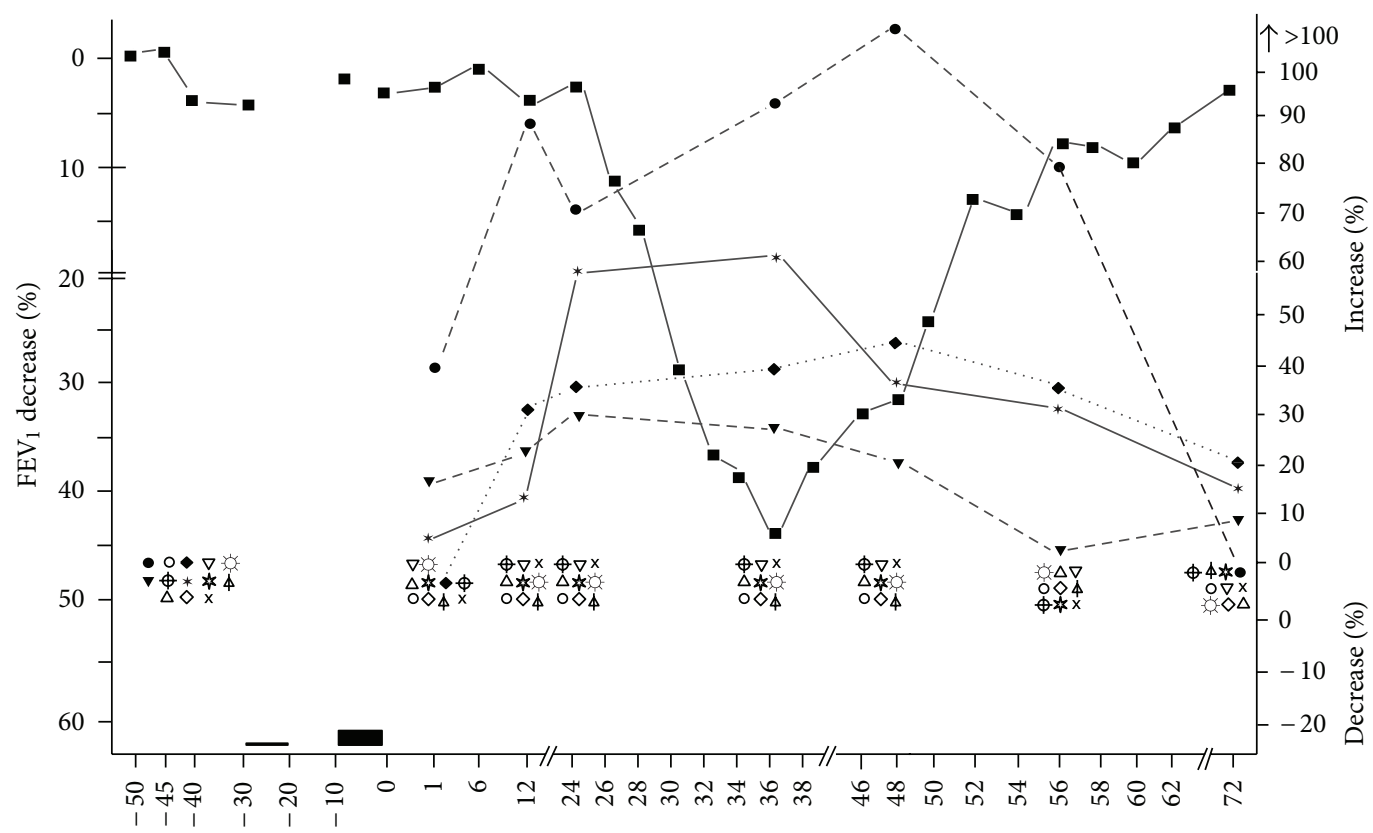

I PBS个 ALL个

Minutes before challenge Hours after challenge

(a)

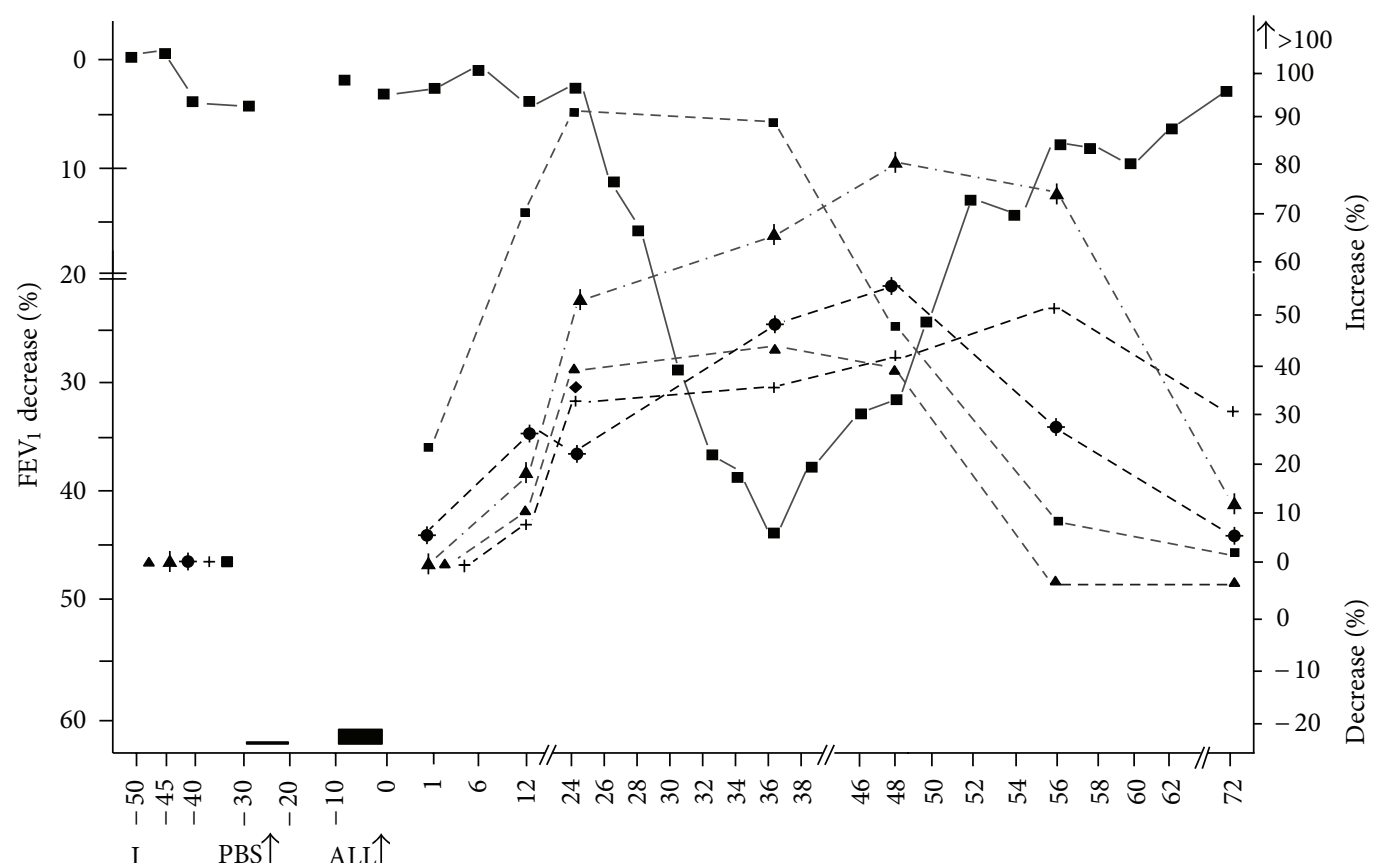

Minutes before challenge Hours after challenge

(a)

○ IL- $1 \beta$

- IL-2

$\Delta$ IL-3

$\diamond \mathrm{IL}-4$

$\nabla$ IL-7
* IL-10

$\times$ IL-12p40

* IFN- $\gamma$

- TNF- $\alpha$

GM-CSF (b)
- IL-8
4 IL- 12 p70
- IL-13
+ IL-18
- TGF- $\beta$

(b)

FIGURE 2: The repeated delayed asthmatic response to allergen challenge (DYAR) and the mean percentage changes in the concentrations of particular cytokines released from isolated blood cells stimulated with PMA; I: initial (baseline) value; ALL: allergen challenge; PBS: phosphate buffered saline. 
stimulation with nonspecific agents, such as PHA, PMA, fMLP, or LPS [9, 13, 14, 17, 21, 25-33, 35].

The papers dealing with changes of the cytokine concentrations in peripheral blood accompanying the particular asthmatic response types caused solely by the allergens inhaled during the BPTs are relatively unique $[21,45,46]$.

Results concerning the changes of particular cytokines in patients with allergic bronchial asthma relatively vary. These variations could be caused by several factors, such as variations in the asthmatic patient populations; different bronchial asthma phenotypes; intermittent or concomitant conditions; unexpected or uncontrolled additional allergen exposure; extent of the diagnostic procedures; differences in the allergen challenge techniques and techniques of material collection and processing; stage of the bronchial asthma and the functional stage of particular cell types in various media at the time of material collection; physiologic variations in the cytokine concentrations in the same medium as well as among the media themselves.

One of the very important aspects and cause of some differences among the results reported by various papers concerns the mode of stimulation of the airway and circulating cells to release their cytokines. The cells stimulated by the "in vivo" inhaled allergen released distinctly lower cytokine amounts than those released after "in vitro" stimulation.The "in vitro" stimulation by a nonspecific but very powerful stimulating agent results usually in a release of the almost complete intracellular cytokine potential, including even those portions which would not be released upon natural allergen inhalation [45]. It can, therefore, be presumed that the cytokine amounts released after an "in vitro" stimulation with nonspecifically acting compounds, such as PHA, PMA, fMLP, or LPS, will be distinctly higher than those released by the natural allergen exposure. This fact may be confirmed by our current results of higher cytokine concentrations released into supernatants after the "in vitro" stimulation with PMA than cytokine amounts recorded at corresponding time intervals in either nonstimulated plasma (Table 6) or nonstimulated serum (Table 4) as well as by our previous results [45]. Moreover, no significant differences were found in the cytokine concentrations between the serum and the plasma (Tables 4 and 6). Unfortunately, the possible discrepancy between the cytokine amounts and ratio released by the "in vivo" inhaled allergen and those released after the "in vitro" stimulation of the isolated BAL or peripheral blood cells by nonspecific stimulating agents has not yet been sufficiently investigated.

An additional aspects concerns the releasing capability of the particular nonspecific agents. Data presented in Table 5 suggest some small, statistically insignificant, differences in the releasing capacity of PHA, PMA, and FMLP. PHA and PMA presented similar stimulating capacity, whereas the cytokine amounts released by fMLP were slightly lower. In a pilot study, data of which are not shown, we have compared the stimulating capacity of these agents in various concentrations. The concentrations used in the present study were most effective in the cytokine release from the isolated blood cells, whereas the higher concentrations were not significantly more effective.
Another important factor influencing the conclusions drawn from various studies is the time schedule of the material collection. The data presented in Table 6 show clearly that the significant changes in the concentrations of particular cytokines, both in plasma and in supernatants, appear 24-56 hours after the allergen challenge, and not before or shortly after the challenge. This fact would stress the importance of repeated recording of the investigated parameters, in this case cytokine concentrations, related to a well-defined and controlled event, such as bronchial allergen challenge. This is the only method to follow the dynamic aspects of the certain parameter changes. The single cytokine determination, related to a certain time interval only, seems to us to be of a limited value and can be accepted only for the screening purposes.

A complicating factor of the cytokine studies is a dearth of comprehensive data concerning the cytokine and chemokine profiles in peripheral blood, BAL fluid, or sputum of healthy subjects, which could be used as reference standards [13, $33,47,48]$. The prechallenge serum as well as supernatant concentrations of cytokines recorded in DYAR patients did not differ significantly from healthy control subjects, whereas they differed from the values measured in patients developing IAR, LAR, or DLAR (Tables 4 and 5). The cytokine profiles recorded during the DYAR [45] differed also from those measured by other investigators in patients developing IAR, LAR, or DLAR $[15,16,18,25]$. These facts would suggest involvement of different immunologic mechanisms in the DYAR and those underlying the other asthmatic response types.

The results of this study are consistent with the results of our previous studies [40,41,44,45], especially with significant changes in the $T h_{1} / \mathrm{Th}_{2}$ ratio in peripheral blood in favour of $\mathrm{Th}_{1}$ cells, increased intracellular concentrations of IFN- $\gamma$ and IL-2, but not those of IL- 4 or IL-5, increased blood leukocyte, neutrophil, and monocyte, but not eosinophil, counts, increased plasma concentrations of $\mathrm{LTB}_{4}$ and MPO, and serum concentration changes of various soluble adhesion molecules during the DYAR.

These results would suggest involvement of the cellmediated hypersensitivity upon participation of $\mathrm{Th}_{1}$ lymphocytes (IL-2, IFN- $\gamma$, GM-CSF, and TGF- $\beta$ ), neutrophils (IL8 , G-CSF, and TNF- $\alpha$ ), monocytes (IL-18, TNF- $\alpha$, and TGF$\beta$ ) and probably also NK cells (IL-2, IFN- $\gamma$, IL-8, G-CSF, GM-CSF, and TGF- $\beta$ ) in the clinical DYAR. An additional role of macrophages (IL-8, IL-13, IL-18, TNF- $\alpha$, GM-CSF, and G-CSF), epithelial and endothelial cells (IL-8, IL-18, and TNF- $\alpha$ ) in the immunologic mechanisms leading to the development of the DYAR cannot be even excluded. However, since most of the cytokines are not exclusive products of only one cell type, but they can usually be produced by various cell types and lineages, the determination of the role of particular cell type by means of cytokine profiles becomes to be relatively complicated issue. Moreover, the number of cytokines investigated was relatively limited and some other, even important, cytokines, such as IL-17, IL10 superfamily, and TNF superfamily members have not been included in the current study, either for technical reasons or with respect to the lack of the suitable commercial 
ELISA kits for these cytokines at the time of this study. This aspect can be considered as a certain deficit of the current study. Nevertheless, more concurrent investigations, including more cytokines, have to be performed to clarify the DYAR and the underlying immunologic mechanisms.

\section{Conflict of Interests}

The author has no conflict of interests to be disclosed.

\section{References}

[1] P. Christodoulopoulos, M. K. Tulic, M. Kontolemas, and Q. Hamid, "Immunopathology of allergic airway inflammation," in Middletons's Allergy, Principles \&Practice, N. F. Adkinson, J. W. Yunginger, W. W. Busse, B. S. Bochner, S. T. Holgate, and F. E. R. Simons, Eds., pp. 501-514, Mosby, St. Louis, Mo, USA, 6th edition, 2003.

[2] S. T. Holgate, R. F. Lemanske, O. 'Byrne PM, and S. Kukumanu, "Asthma pathogenesis," in Middleton's Allergy, Principles and Practice, N. F. Adkinson, B. S. Bochner, W. W. Busse et al., Eds., pp. 893-919, Mosby-Elsevier, Philadelphia, Pa, USA, 7th edition, 2009.

[3] S. P. Peters, "Heterogeneity in the pathology and treatment of asthma," American Journal of Medicine, vol. 115, no. 3, pp. 4954, 2003.

[4] R. H. Green, C. E. Brightling, and P. Bradding, "The reclassification of asthma based on subphenotypes," Current Opinion in Allergy and Clinical Immunology, vol. 7, no. 1, pp. 43-50, 2007.

[5] K. K. Sheth and R. F. Lemanske, "The early and late asthmatic response to allergen challenge," in Asthma and Rhinitis, W. W. Busse and S. T. Holgate, Eds., pp. 946-960, Blackwell Scientific Publications, Oxford, UK, 1995.

[6] L. Borish and L. J. Rosenwasser, "Cytokines in allergic inflammation," in Middleton's Allergy, Principlea and Practice, N. F. Adkinson, B. S. Bochner, W. W. Busse, S. T. Holgate, R. F. Lemanske, and F. E. Simons, Eds., pp. 165-179, Mosby-Elsevier, Philadelphia, Pa, USA, 7th edition, 2009.

[7] A. M. Bentley, A. B. Kay, and S. R. Durham, "Human late asthmatic responses," in Allergy and Allergic Diseases, A. B. Kay, Ed., pp. 1113-1130, Blackwell Scientific Publications, Oxford, UK, 1997.

[8] N. N. Jarjour, W. J. Calhoun, E. A. B. Kelly, G. J. Gleich, L. B. Schwartz, and W. W. Busse, "The immediate and late allergic response to segmental bronchopulmonary provocation in asthma," American Journal of Respiratory and Critical Care Medicine, vol. 155, no. 5, pp. 1515-1521, 1997.

[9] L. Y. Liu, S. K. Mathur, J. B. Sedgwick, N. N. Jarjour, W. W. Busse, and E. A. B. Kelly, "Human airway and peripheral blood eosinophils enhance Th1 and Th2 cytokine secretion," Allergy, vol. 61, no. 5, pp. 589-597, 2006.

[10] Z. Pelikan, M. Pelikan, M. Kruis, and M. P. F. Berger, "The immediate asthmatic response to allergen challenge," Annals of Allergy, vol. 56, no. 3, pp. 252-260, 1986.

[11] Z. Pelikan and M. Pelikan-Filipek, "The late asthmatic response to allergen challenge-part I," Annals of Allergy, vol. 56, no. 5, pp. 414-420, 1986.

[12] N. A. Lee, E. W. Gelfand, and J. J. Lee, "Pulmonary T cells and eosinophils: coconspirators or independent triggers of allergic respiratory pathology?" Journal of Allergy and Clinical Immunology, vol. 107, no. 6, pp. 945-957, 2001.
[13] C. K. Wong, C. Y. Ho, F. W. S. Ko et al., "Proinflammatory cytokines (IL-17, IL-6, IL-18 and IL-12) and Th cytokines (IFN- $\gamma$, IL-4, IL-10 and IL-13) in patients with allergic asthma," Clinical and Experimental Immunology, vol. 125, no. 2, pp. 177-183, 2001.

[14] T. Shirai, K. Suzuki, N. Inui, T. Suda, K. Chida, and H. Nakamura, "Th1/Th2 profile in peripheral blood in atopic cough and atopic asthma," Clinical and Experimental Allergy, vol. 33, no. 1, pp. 84-89, 2003.

[15] W. C. Moore, J. D. Hasday, S. S. Meltzer, P. L. Wisnewski, B. White, and E. R. Bleecker, "Subjects with mild and moderate asthma respond to segmental allergen challenge with similar, reproducible, allergen-specific inflammation," Journal of Allergy and Clinical Immunology, vol. 108, no. 6, pp. 908-914, 2001.

[16] G. Hansen, G. Berry, R. H. DeKruyff, and D. T. Umetsu, "Allergen-specific Th 1 cells fail to counterbalance Th2 cellinduced airway hyperreactivity but cause severe airway inflammation," Journal of Clinical Investigation, vol. 103, pp. 175-183, 1999.

[17] J. M. Smart and A. S. Kemp, "Increased Th1 and Th2 allergeninduced cytokine responses in children with atopic disease," Clinical and Experimental Allergy, vol. 32, no. 5, pp. 796-802, 2002.

[18] K. Matsumoto, G. M. Gauvreau, T. Rerecich, R. M. Watson, L. J. Wood, and P. M. O'Byrne, "Il-10 production in circulating $\mathrm{T}$ cells differs between allergen-induced isolated early and dual asthmatic responders," Journal of Allergy and Clinical Immunology, vol. 109, no. 2, pp. 281-286, 2002.

[19] Z. Pelikan, M. Pelikan-Filipek, and J. Oers van, "The early asthmatic response to allergen challenge (EAR) and its pharmacologic modulation," Allergy, vol. 56, supplement 68, abstarct 66, p. 24, 2001.

[20] Z. Pelikan, "The effects of various inhaled drugs on the late asthmatic response (LAR) to the bronchial challenge with allergens," Allergy, vol. 56, supplement 68, p. 25, 2001.

[21] M. Yoshida, R. M. Watson, T. Rerecich, and P. M. O’Byrne, "Different profiles of T-cell IFN- $\gamma$ and IL-12 in allergen-induced early and dual responders with asthma," Journal of Allergy and Clinical Immunology, vol. 115, no. 5, pp. 1004-1009, 2005.

[22] P. Gosset, A. Tsicopoulos, B. Wallaert et al., "Increased secretion of tumor necrosis factor $\alpha$ and interleukin- 6 by alveolar macrophages consecutive to the development of the late asthmatic reaction," Journal of Allergy and Clinical Immunology, vol. 88, no. 4, pp. 561-571, 1991.

[23] C. Gratziou, M. Carroll, S. Montefort, L. Teran, P. H. Howarth, and S. T. Holgate, "Inflammatory and T-cell profile of asthmatic airways 6 hours after local allergen provocation," American Journal of Respiratory and Critical Care Medicine, vol. 153, no. 2, pp. 515-520, 1996.

[24] J. Bettiol, J. Sele, M. Henket et al., "Cytokine production from sputum cells after allergenic challenge in IgE-mediated asthma," Allergy, vol. 57, no. 12, pp. 1145-1150, 2002.

[25] K. J. Bodey, A. E. Semper, A. E. Redington et al., "Cytokine profiles of BAL T cells and T-cell clones obtained from human asthmatic airways after local allergen challenge," Allergy, vol. 54, no. 10, pp. 1083-1093, 1999.

[26] M. Majori, M. Corradi, A. Caminati, G. Cacciani, S. Bertacco, and A. Pesci, "Predominant TH1 cytokine pattern in peripheral blood from subjects with chronic obstructive pulmonary disease," Journal of Allergy and Clinical Immunology, vol. 103, no. 3, pp. 458-462, 1999. 
[27] L. Liu, N. N. Jarjour, W. W. Busse, and E. A. B. Kelly, "Enhanced generation of helper $\mathrm{T}$ type 1 and 2 chemokines in allergeninduced asthma," American Journal of Respiratory and Critical Care Medicine, vol. 169, no. 10, pp. 1118-1124, 2004.

[28] E. A. Becky Kelly, R. R. Rodriguez, W. W. Busse, and N. N. Jarjour, "The effect of segmental bronchoprovocation with allergen on airway lymphocyte function," American Journal of Respiratory and Critical Care Medicine, vol. 156, no. 5, pp. 14211428, 1997.

[29] J. C. Virchow, C. Walker, D. Hafner et al., "T cells and cytokines in bronchoalveolar lavage fluid after segmental allergen provocation in atopic asthma," American Journal of Respiratory and Critical Care Medicine, vol. 151, no. 4, pp. 960-968, 1995.

[30] V. Batra, A. I. Musani, A. T. Hastie et al., "Bronchoalveolar lavage fluid concentrations of transforming growth factor (TGF)- $\beta 1$, TGF- $\beta 2$, interleukin (IL)- 4 and IL-13 after segmental allergen challenge and their effects on $\alpha$-smooth muscle actin and collagen III synthesis by primary human lung fibroblasts," Clinical and Experimental Allergy, vol. 34, no. 3, pp. 437-444, 2004.

[31] N. Krug, J. Madden, A. E. Redington et al., "T-cell cytokine profile evaluated at the single cell level in BAL and blood in allergic asthma," American Journal of Respiratory Cell and Molecular Biology, vol. 14, no. 4, pp. 319-326, 1996.

[32] M. P. Hallsworth, C. P. C. Soh, H. J. Lane, E. P. Arm, and M. H. Lee, "Selective enhancement of GM-CSF,TNF- $\alpha$, lL-1 $\beta$ andIL8 production by monocytes and macrophages of asthmatic subjects," European Respiratory Journal, vol. 7, no. 6, pp. 10961102, 1994.

[33] T. Terashima, K. Amakawa, A. Matsumaru, S. Van Eeden, J. C. Hogg, and K. Yamaguchi, "BAL induces an increase in peripheral blood neutrophils and cytokine levels in healthy volunteers and patients with pneumonia," Chest, vol. 119, no. 6, pp. 1724-1729, 2001.

[34] S. J. Till, S. R. Durham, K. Rajakulasingam et al., "Allergeninduced proliferation and interleukin-5 production by bronchoalveolar lavage and blood $\mathrm{T}$ cells after segmental allergen challenge," American Journal of Respiratory and Critical Care Medicine, vol. 158, no. 2, pp. 404-411, 1998.

[35] V. Brown, T. J. Warke, M. D. Shields, and M. Ennis, "T cell cytokine profiles in childhood asthma," Thorax, vol. 58, no. 4 , pp. 311-316, 2003.

[36] D. T. Umetsu, O. Akbari, and R. H. DeKruyff, "Regulatory T cells control the development of allergic disease and asthma," Journal of Allergy and Clinical Immunology, vol. 112, no. 3, pp. 480-487, 2003.

[37] A. O. Magnan, L. G. Mély, C. A. Camilla et al., "Assessment of the Th1/Th2 paradigm in whole blood in atopy and asthma: increased IFN- $\gamma$-producing $\mathrm{CD}^{8+} \mathrm{T}$ cells in asthma," American Journal of Respiratory and Critical Care Medicine, vol. 161, no. 6, pp. 1790-1796, 2000.

[38] H. H. Kariyawasam, M. Aizen, J. Barkans, D. S. Robinson, and A. B. Kay, "Remodeling and airway hyperresponsiveness but not cellular inflammation persist after allergen challenge in asthma," American Journal of Respiratory and Critical Care Medicine, vol. 175, no. 9, pp. 896-904, 2007.

[39] T. Shirai, N. Inui, T. Suda, and K. Chida, "Correlation between peripheral blood T-cell profiles and airway inflammation in atopic asthma," Journal of Allergy and Clinical Immunology, vol. 118, no. 3, pp. 622-626, 2006.
[40] Z. Pelikan, M. Pelikan-Filipek, and J. H. Oostenbrink, "Delayed asthmatic response (DYAR), its clinical features and pharmacologic modulation," Journal of Allergy and Clinical Immunology, vol. 99, no. 1, article 321, 1997.

[41] Z. Pelikan, "Delayed-type asthmatic response to bronchial challenge with allergen, I: clinical features," Annals of Allergy, Asthma and Immunology, vol. 104, no. 5, pp. 394-404, 2010.

[42] G. Melillo, S. Bonini, G. Cocco et al., "EAACI provocation tests with allergens. Report prepared by the European Academy of Allergology and Clinical Immunology Subcommittee on provocation tests with allergens," Allergy, vol. 52, no. 35, pp. 135, 1997.

[43] D. J. Houd and C. C. Taylor, Multivariate Analysis of Variance and Repeated Measures, Chapman and Hall, London, UK, 1987.

[44] Z. Pelikan, "Delayed asthmatic response to bronchial challenge with allergen-mediators, eicosanoids, eosinophil and neutrophil constituents in the blood and urine," Respiration, vol. 82, pp. 225-236, 2011.

[45] Z. Pelikan, "Delayed type of asthmatic response to allergen challenge and cytokines in the peripheral blood," Respiration, vol. 84, no. 5, pp. 385-395, 2012.

[46] T. Kawayama, P. M. O'Byrne, R. M. Watson et al., "Effects of inhaled ciclesonide on circulating T-helper type 1/T-helper type 2 cells in atopic asthmatics after allergen challenge," Clinical and Experimental Allergy, vol. 36, no. 11, pp. 1417-1424, 2006.

[47] C. Härtel, N. Adam, T. Strunk, P. Temming, M. MüllerSteinhardt, and C. Schultz, "Cytokine responses correlate differentially with age in infancy and early childhood," Clinical \& Experimental Immunology, vol. 142, pp. 446-453, 2005.

[48] M. Halonen, I. C. Lohman, D. A. Stern et al., "Th1/Th2 patterns and balance in cytokine production in the parents and infants of a large birth cohort," Journal of Immunology, vol. 182, no. 5, pp. 3285-3293, 2009. 


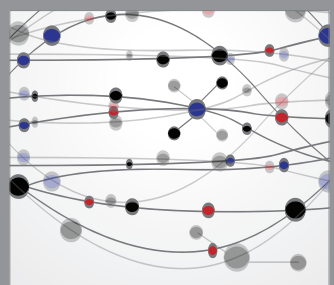

The Scientific World Journal
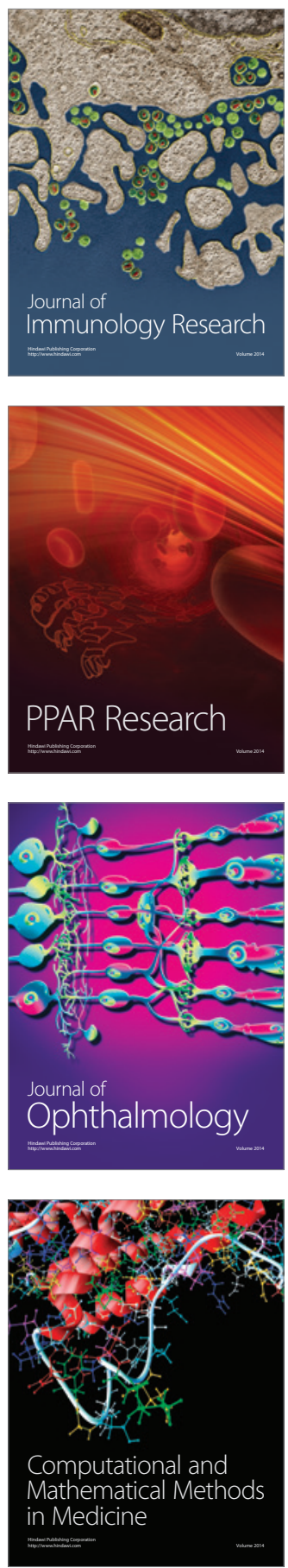

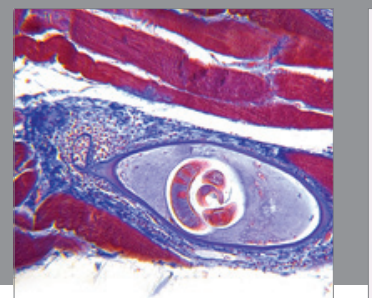

Gastroenterology

Research and Practice
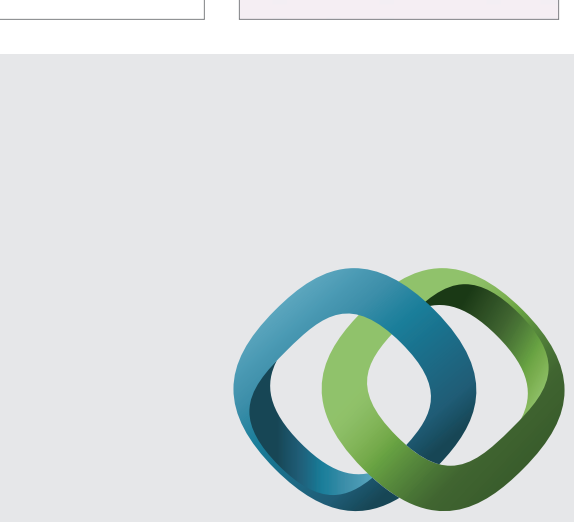

\section{Hindawi}

Submit your manuscripts at

http://www.hindawi.com
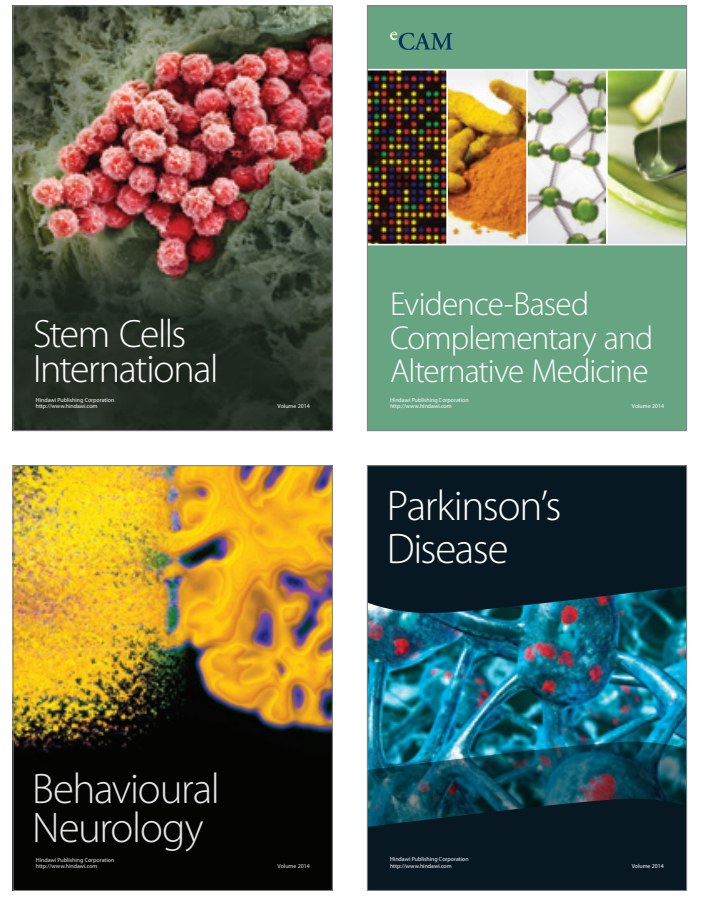
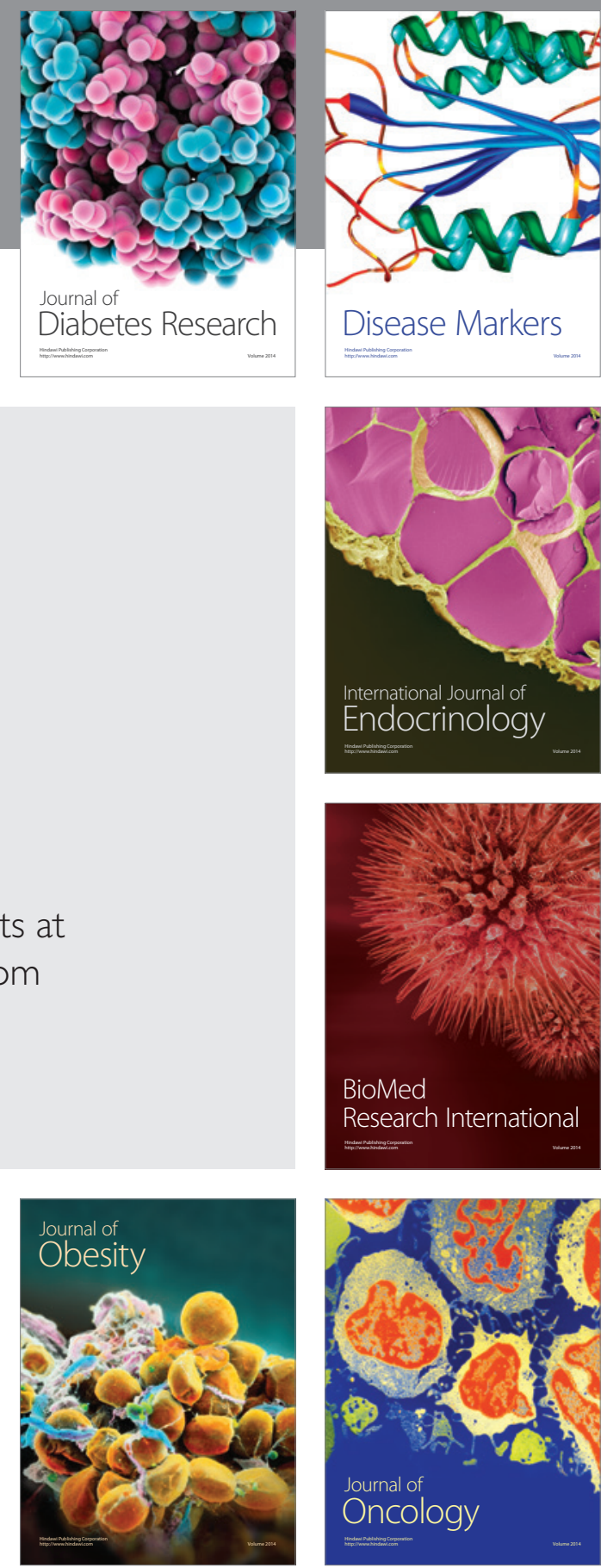

Disease Markers
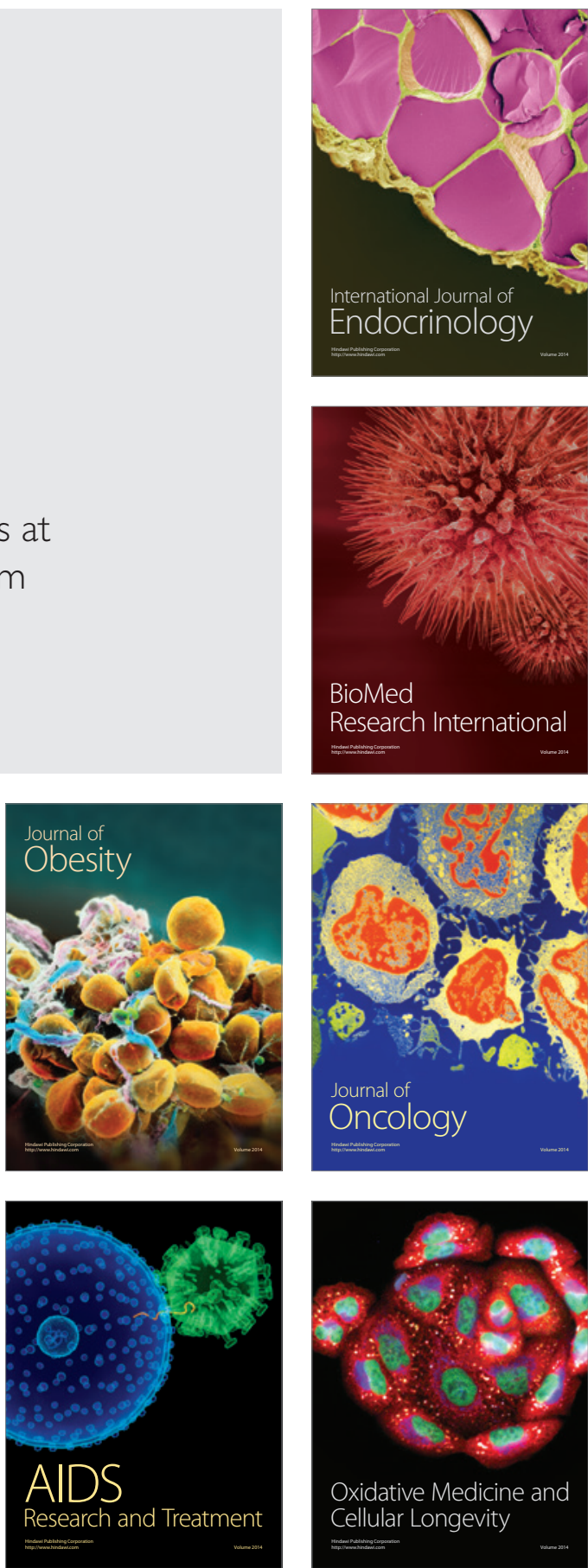